\title{
Measures of perceived linear size, sagittal motion, and visual angle from optical expansions and contractions
}

\author{
WALTER C. GOGEL \\ University of Califormia, Santa Barbara, Califormia \\ and \\ DAVID W. EBY \\ University of Michigan, Ann Arbor, Michigan
}

\begin{abstract}
Using monocular observation, open-loop measurements were obtained of the perceptions of linear size, angular size, and sagittal motion associated with the terminal (largest or smallest) stimuli of repetitive optical expansions and contractions using 1-D or 2-D displays produced on a video monitor at a constant distance from the observer. The perceptions from these dynamic conditions were compared with those from static conditions in which the stimuli were of the same physical size and at the same physical distance as the terminal dynamic stimuli, but that were not part of the optical expansions or contractions. One result, as expected, was that the measures of perceived linear and angular size differed, but also, unexpectedly, some substantial errors were associated with the measures of perceived angular size. Another result was that the amount of size constancy was considerably less than was expected from the obtained amount of perceived motion in depth. Consistent with the latter result, it was found that the size-distance invariance hypothesis (SDIH), using the physical visual angles of the terminal stimuli, predicted only about half of the perceived motion in depth obtained with the dynamic changes. Using the obtained measures of perceived visual angles in the SDIH increased rather than decreased the error in predicting the amount of motion in depth as perceived. An additional experiment suggests that at least some of the error in the measurement of the perceived visual angle is a consequence of error in the perceived origin of the visual angles. The absence of the expected relation between size constancy and perceived motion in depth in the dynamic conditions is hypothesized to be due to cognitive processes associated with off-sized perceptions of the stimuli.
\end{abstract}

A retinal image of constant physical shape that expands or contracts symmetrically can produce the perception of a stimulus object moving toward or away from the observer. This perception can occur whether an object of constant physical size is actually physically moving sagittally, or whether a physical motion in depth is being simulated by a monocularly viewed expanding or contracting stimulus, generated on the screen of a display monitor, and located at a constant distance from the observer in an otherwise dark surround. In addition, the perception of motion in depth from the simulation is often assumed to occur even though the only variable available to determine both the perceived sizes of the stimuli and the perceived sagittal

The preparation of this article was supported by Research Grant $\mathrm{MH}$ 39457 from the National Institute of Mental Health to W.C.G. The research was conducted at the University of California, Santa Barbara. The authors thank Jerome D. Tietz for his help in the analysis of the data throughout the study, for building the apparatus and collecting the data of Experiment 3, and for his suggestions on a previous draft. We also thank John M. Foley for his helpful discussions of related theoretical issues. In a previous article (Gogel, 1990), the present study is referred to as W. C. Gogel and D. W. Eby, The perception of visual angle in optical change. Unpublished manuscript. Correspondence should be addressed to W. C. Gogel, Department of Psychology, University of California, Santa Barbara, CA 93106 (gogel@psych.ucsb.edu). motion is the changing size of the stimulus on the eye. This assumption requires some explanation. It is usually accepted that the amount of depth perceived in either the real or the simulated motion is inversely related to the amount of change in the perceived linear size of the expanding or contracting stimulus. This is illustrated by two extremes in the perceptions that can occur. In one extreme, it is expected, if the stimulus is perceived as expanding or contracting in linear size proportional to its angular expansion or contraction on the eye, that there will be no perception of motion in depth. At the other extreme, if the same stimulus is perceived as remaining a constant linear size (despite its changing visual angle on the monitor and eye), then the motion in depth will be perceived at a maximum for a particular perceived starting size of the stimulus (i.e., the full simulated motion in depth will be perceived). Between these extremes, any number of other perceptions of linear size change and motion in depth are possible, depending on how the angular (optical) change is proportioned (divided) between the perceptions of linear size and distance. For example, if only part of the optical change on the eye is involved in achieving the perception of linear size, the amount of perceived sagittal motion, although possibly remaining substantial, would be less than that simulated. The amount of change 
in the perceived linear size of the stimulus given the size changes on the eye can be expressed as an amount of size constancy. In this study, size constancy is designated as complete or equal to 1.00 (perfect size constancy) if the ratio of the perceived linear sizes at the terminal (farthest and nearest) perceptions of motion in depth of an expansion or contraction is unity and is designated as zero (no size constancy) if this ratio is equal to the ratio of the retinal sizes of the stimuli. The less the change in retinal size that is applied to perceiving changes in linear size, the greater the change in retinal size available for producing a perceived motion of the stimulus in depth. This assumed tradeoff between allocating changes in the retinal size of the stimulus to changes in perceived motion in depth and to changes in perceived size can be predicted by the size-distance invariance hypothesis (SDIH), which is as follows:

$$
S^{\prime} / D^{\prime}=2 \tan (\theta / 2),
$$

where at any instant of time $S^{\prime}, D^{\prime}$, and $\theta$ are the perceived linear size, perceived egocentric distance, and physical visual angle of the stimulus, respectively. ${ }^{1}$ According to Equation 1, the perceived motion in depth, $d^{\prime}$, between the farthest $D_{\mathrm{f}}^{\prime}$ and nearest $D_{\mathrm{n}}^{\prime}$ perceived distances of the stimulus resulting from the optical size change (expansion or contraction) is

$$
d^{\prime}=D_{\mathrm{f}}^{\prime}-D_{\mathrm{n}}^{\prime}=S_{\mathrm{s}}^{\prime} / 2 \tan \left(\theta_{\mathrm{s}} / 2\right)-S_{\mathrm{L}}^{\prime} / 2 \tan \left(\theta_{\mathrm{L}} / 2\right),
$$

where $S_{\mathrm{s}}^{\prime}$ and $S_{\mathrm{L}}^{\prime}$ are the perceived linear sizes associated with the smallest (perceptually farthest) and largest (perceptually nearest) stimuli, and $\theta_{\mathrm{s}}$ and $\theta_{\mathrm{L}}$ are the smallest and largest physical visual angles of the stimulus, respectively. Throughout this paper, as is indicated in this equation, the primed notation refers to perceived and the unprimed notation to the physical characteristics of the stimulus.

According to Equation 2, given the values of the physical terminal visual angles $\left(\theta_{\mathrm{s}}\right.$ and $\left.\theta_{\mathrm{L}}\right)$ of an optical expansion and contraction, $d^{\prime}$ will be determined by the changes in the perceived linear sizes, $S_{\mathrm{s}}^{\prime}$ and $S_{\mathrm{L}}^{\prime}$, occurring between the terminal values of the stimuli. Thus, Equation 2 might be expected to predict the perceived sagittal depth, $d^{\prime}$, whether the stimulus is an object of physically constant size that is physically moving toward or away from the observer, or the same changes in angular size are produced on a physical display monitor located at a constant distance from the observer. The latter case, as contrasted with the former, however, allows for the ready elimination of all factors of changing distance other than the factor of the changes in visual angle to produce the motion in perceived depth. In the extreme case in which all of the change in visual angle is used by the visual system to produce only changes in perceived size, the perception of motion in depth, $d^{\prime}$, according to the SDIH, will be zero throughout the optical size change. Thus, according to Equation 2, no perception of motion in depth is expected if the perception of linear size is proportional to the perception of angular size - that is, $d^{\prime}$ equals zero only when at the terminal positions of the optical change $S_{\mathrm{s}}^{\prime} / S_{\mathrm{L}}^{\prime}=$ $2 \tan \left(\theta_{\mathrm{s}} / 2\right) / 2 \tan \left(\theta_{\mathrm{L}} / 2\right)$. In the second extreme case, all of the change in visual angle is used by the visual system to produce only perceived motion in depth with none remaining to produce changes in perceived size. In the latter case, according to the SDIH as expressed by Equation 2, perfect or complete size constancy will be present (i.e., $S_{\mathrm{s}}^{\prime} / S_{\mathrm{L}}^{\prime}=1$ ) throughout the optical change with a resulting robust perception of motion in depth. Results intermediate between these extremes also might be expected from the SDIH as expressed by Equation 2. Thus, the perceived motion in depth is expected to be predicted from Equation 2 in all cases in which the amount of size constancy varies from zero to perfect constancy.

The SDIH often predicts with considerable success the relation between the perception of size and distance, when effective cues of changing distance are present in addition to changes in retinal size. However, as is the case in the present study, under conditions in which only changes in retinal size are available, the predictive ability of Equation 2 probably, at best, is limited. For example, in a study by Swanston and Gogel (1986, Experiments 1 and 3 ), a repetitively expanding or contracting luminous line at a physically constant distance was monocularly viewed in an otherwise dark visual field. Substantial amounts of perceived sagittal motion were obtained even though the ratio of the perceived linear sizes of the line at the large and small extremes of visual angle were similar to the tangents of the visual angles at these extremes.

An examination of the perceptions associated with an optical size change, whether caused by an object physically moving relative to the observer or a simulation on a monitor of such a motion, and whether or not optical size change is the only cue available, requires, consistent with Equation 2, that the observers differentiate between perceived linear and perceived angular size. A procedure used in the present study to accomplish this is shown in Figure 1, in which a stimulus of a physical linear size $S$ and a physical visual angle $\theta$ are located at a physical distance $D$ from the observer with the perceived linear size labeled $S^{\prime}$ and perceived angular size labeled $\theta^{\prime}$. Both of these kinds of perceptual measures were obtained using unseen posts and hands (open-loop conditions) by adjusting the lateral separation between a pair of posts located within arm's reach at three different physical distances $\left(D_{\mathrm{N}}, D_{\mathrm{M}}\right.$, or $\left.D_{\mathrm{F}}\right)$, as indicated in Figure 1 by the three pairs of filled squares. Each post was independently movable laterally by the observer, with one post adjusted by the right hand and the other by the left hand. The adjustments are made by kinesthesis with both the posts and hands unseen by the observer during the adjustments. To measure the perceived linear size (Figure 1A), the observer separated the pair of posts laterally to duplicate the perceived linear width of the stimulus as measured with the alternately presented pair of posts at each of the three different distances of the posts. To measure perceived angular size (Figure 1B), the observer, using the same physical distances of the posts, adjusted the separation of the 


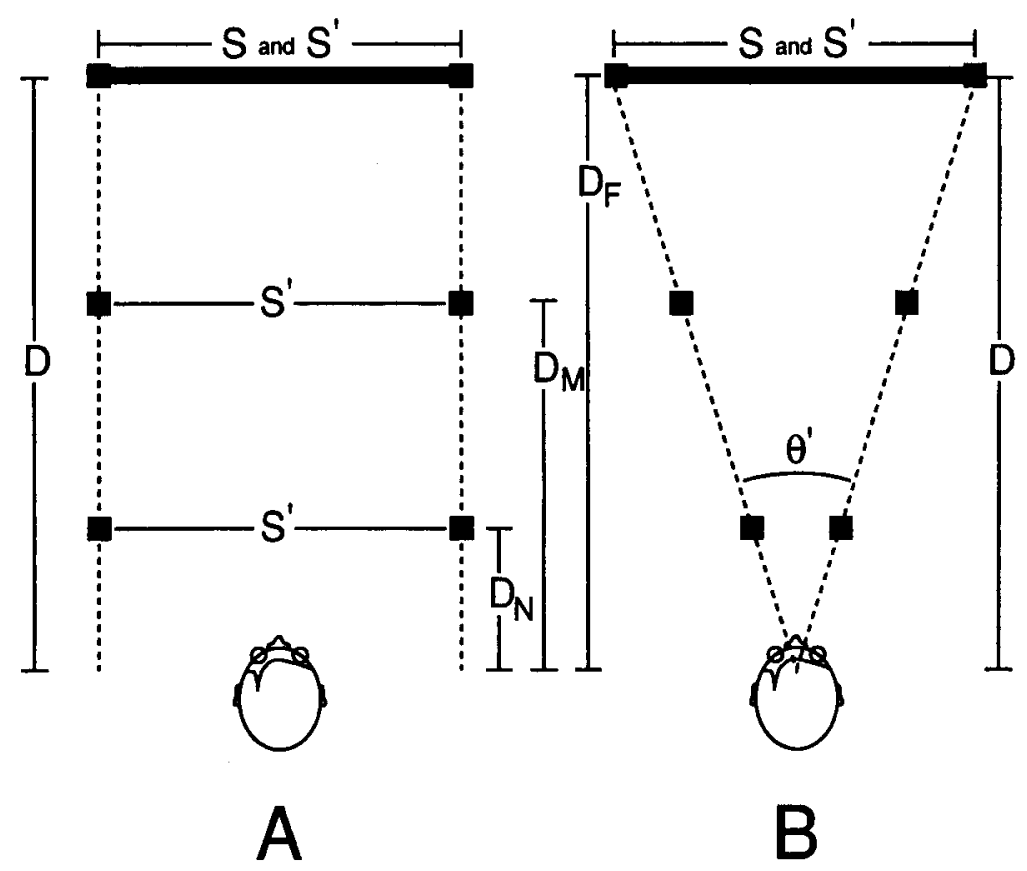

Figure 1. A procedure for distinguishing between the perception of linear size $\left(S^{\prime}\right)$ and the perception of angular size $\left(\theta^{\prime}\right)$ of a stimulus. In both Situations $A$ and $B$, a pair of unseen square posts is presented successively at a near $(\mathrm{N})$, middle $(\mathrm{M})$, or far (F) distance from the observer. Using the open-loop procedure, the posts are laterally adjusted kinesthetically to indicate the perceived linear width (Figure 1A) or the perceived difference in direction to the two ends of the stimulus (Figure 1B) at each distance of the posts. From these adjustments, $S^{\prime}$ and $\theta^{\prime}$ are determined at each of the post distances. It was expected that the post adjustments would be essentially constant, as shown in Figure $1 \mathrm{~A}$, and would increase with each increase in their physical distance from the observer, as shown in Figure $1 \mathrm{~B}$.

pair of posts laterally at each of the three distances of the posts so that the posts of each pair were perceived to be along imaginary lines extending from the observer to each end of the stimulus. As shown in Figure 1 A, the adjusted lateral separation of the posts was expected to be independent of the physical distance of the pair of posts from the observer when the observer indicated perceived linear size. For the perceived angular size adjustments, as illustrated in Figure 1B, unlike the perceived linear size adjustments of the posts in Figure 1A, the adjusted lateral separation of the posts was expected to increase as the physical distance of the pair of posts from the observer increased. Thus, the differences in the post adjustments obtained at the different distances in Figure $1 \mathrm{~A}$ as compared with those in Figure 1B were expected to reflect the distinction between perceived linear and perceived angular size. In both cases, however, it was expected that the measurement of $S^{\prime}$ and $\theta^{\prime}$, using the adjusted separation of the posts, would be independent of the post distances.

There are several circumstances that might explain the failure of Equation 2, as found by Swanston and Gogel (1986), to predict the perceived motion in depth obtained from an optical size change. One is that when asked to indicate the perceived linear size of the stimulus, the observers might mistakenly have indicated its perceived an- gular size. If this had happened, it would have been concluded erroneously that the ratios of the perceived linear and angular sizes were equal, suggesting, from Equation 2 of the SDIH, that the perception of sagittal motion, $d^{\prime}$, contrary to the obtained results, should have been zero. Although Swanston and Gogel argued against this interpretation, there clearly is a need in a variety of situations to distinguish between these two kinds of responses to size (see Ono, 1970; Rock, 1983). A second possible concern in applying Equation 2 to the prediction of $d^{\prime}$ in an optical expansion or contraction is that the perceived visual angle, $\theta^{\prime}$, rather than the physical visual angle, $\theta$, might be the proper term to use in making predictions from the SDIH (Gogel \& Da Silva, 1987; Higashiyama, 1992; McCready, 1965, 1986). In this case, Equations 1 and 2 become, respectively,

$$
S^{\prime} / D^{\prime}=2 \tan \left(\theta^{\prime} / 2\right)
$$

and

$$
d^{\prime}=D_{\mathrm{f}}^{\prime}-D_{\mathrm{n}}^{\prime}=S_{\mathrm{s}}^{\prime} / 2 \tan \left(\theta_{\mathrm{s}}^{\prime} / 2\right)-S_{\mathrm{L}}^{\prime} / 2 \tan \left(\theta_{\mathrm{L}}^{\prime} / 2\right) \text {. }
$$

For example, for the same obtained values of $S_{\mathrm{s}}^{\prime}$ and $S_{\mathrm{L}}^{\prime}$, the perceived motion in depth predicted from Equation 4 will be greater than that predicted from Equation 2 if the ratio of $\theta_{\mathrm{s}}^{\prime} / \theta_{\mathrm{L}}^{\prime}$ is less than $\theta_{\mathrm{s}} / \boldsymbol{\theta}_{\mathrm{L}}$. Also, the generality of 
the failure of the SDIH to predict the obtained perception of motion in depth from the optical expansion used in Experiments 1 and 3 of the study by Swanston and Gogel (1986) can be questioned in that, in the Swanston and Gogel study, a 1-D rather than a 2-D stimulus was used in the optical change (see Hershenson, 1992a, 1992b). This possibility is considered in the present study.

\section{EXPERIMENT 1}

The objectives of Experiment 1 were to (1) measure, using the unseen posts and hands, the perceptions of linear size, angular size, and sagittal depth associated with a repetitive, continuous, 2-D optical change, starting first as either an optical expansion or an optical contraction; (2) to determine whether the SDIH as expressed by Equation 2 is able to predict the perception of motion in depth $\left(d^{\prime}\right)$ resulting from these optical changes; and (3) to examine whether deficiencies in applying Equation 2 to the prediction of $d^{\prime}$, if these deficiencies occur, are reduced or eliminated by using the perceived values of visual angle as in Equation 4, rather than the physical values of visual angle as in Equation 2.

Method
Observers
Twelve graduate students ( 6 men and 6 women) from the psy-
chology department were paid observers in the experiment. ${ }^{2}$ All
had a normal acuity $(20 / 20)$ in their right (viewing) eye for near and
far vision as measured using the Keystone orthoscope, and all were
naive concerning the purposes of the experiment.

\section{Apparatus}

Visual alley and measuring posts. The experimental alley in which the stimulus was located was $36 \mathrm{~cm}$ wide and $139 \mathrm{~cm}$ long. An occluder was located at $28 \mathrm{~cm}$ from the observer. The occluder extended across the visual alley and contained an aperture $(27 \mathrm{~cm}$ high and $33 \mathrm{~cm}$ wide) that could be opened or closed remotely by the experimenter from a position outside the alley in order to reveal or occlude the observer's view of the stimulus. The stimulus was displayed on a monitor, the front (display) surface of which was always $40 \mathrm{~cm}$ from the observer's eye. The observation booth contained a chin cup, adjustable in height, which allowed the experimenter to position the eyes of each observer at the same height as the center of the stimulus ( $19.5 \mathrm{~cm}$ above the alley floor). The shape of the chin cup was such that the observer, although restricted from moving the head laterally, could rotate the head around a vertical axis at the position of the chin. An adaptation surface $\left(18.5 \mathrm{~cd} / \mathrm{m}^{2}\right)$ was located on the left wall of the observation booth. This surface, when illuminated, light-adapted the observer between trials with the viewing aperture closed. The visual alley and aperture surface were covered with black velveteen. The only object visible during a trial was the stimulus presented in an otherwise completely dark alley with the aperture and the observation booth also completely dark during the observations.

At $9.3 \mathrm{~cm}$ below the floor of the alley were two small knobs, one on the top of each of two vertical square metal posts. The metal posts ( $1.25 \mathrm{~cm}$ in cross section and $10 \mathrm{~cm}$ high) could be independently moved laterally in opposite or in the same directions by the observer by grasping one post in each hand with a thumb on each knob. These laterally, kinesthetically adjustable posts, to be used in the measurement of both the perceived linear size and perceived angular size of the stimuli, as shown in Figure 1, could be pinned by the experimenter at one of the three distances from the observer so that their sagittal motion was completely restricted without restricting their lateral adjustment. The lateral separation between the posts was read from a double-sliding ruler by the experimenter from a position outside the alley. When the two posts were brought together laterally and were unpinned, they could be kinesthetically moved sagittally as a pair toward or away from the observer. Their movement toward the observer was stopped at a distance of $15.7 \mathrm{~cm}$ from the observer by the far surface of a stationary third post. The sagittal separation between the most adjacent surfaces of this physically stationary post and the laterally joined pair of sagittally movable posts was adjustable kinesthetically by the observer. This permitted the observer to indicate the amount of perceived motion in depth produced by the cycles of continuous optical change in size of the stimulus between the two perceived terminal distances. The observer's sagittal depth adjustments of the posts were measured by means of a variable potentiometer geared to the posts and were displayed by a computer on a second monitor screen outside the alley that was visible only to the experimenter. When making either the lateral or sagittal adjustments of the posts, the observer was unable to see either the posts or his/her hands; that is, throughout this study, all the measures obtained, whether of perceived linear size, perceived visual angle, or perceived sagittal motion, were open-loop adjustments.

The stimuli. All stimuli were presented on a Conrac black-andwhite video monitor (screen height $48 \mathrm{~cm}$ and width $36 \mathrm{~cm}$ ) that remained at a constant physical distance of $40 \mathrm{~cm}$ from the observer's eye. The viewing throughout the study was with the right eye only, with the left eye occluded by an eye patch. A red filter covered the screen of the monitor in order to reduce the visibility of the phosphor trace and thus the persistence of the stimulus as it changed size on the monitor.

The stimuli were computer-generated squares consisting of luminous outlines approximately $1.5 \mathrm{~mm}$ wide. Two of the stimulus conditions were dynamic and two were static. The dynamic conditions consisted of cycles of a physically expanding and contracting square generated on the monitor. The terminal stimuli of the continuous optical change in the dynamic conditions occurred repeatedly between a square stimulus of a size of $7.0 \mathrm{~cm}$ and another of $23.3 \mathrm{~cm}$ on a side. Either an expansion or a contraction occurred first. In the expansion-first condition, the size started at $7.0 \mathrm{~cm}$, expanded to $23.3 \mathrm{~cm}$, and then contracted to $7.0 \mathrm{~cm}$, with this cycle repeated. For the contraction-first condition, the size started at $23.3 \mathrm{~cm}$, contracted to $7.0 \mathrm{~cm}$, and then expanded to $23.3 \mathrm{~cm}$, with this cycle repeated. The duration for a half-cycle of motion was $4.7 \mathrm{sec}$. A terminal stimulus of the dynamic conditions remained stationary on the display for $1 \mathrm{sec}$ before the direction of the size change was reversed. The dynamic stimuli were created using animation software developed by Loomis and Eby (1987) in which the simulation was of an object of constant size and velocity moving sagittally with respect to the observer's right eye. The static conditions consisted of squares of unchanging (static) size presented on the display screen at $40.0 \mathrm{~cm}$ prior to presenting the dynamic condition, which started at the same size and distance. Thus, a $7.0-\mathrm{cm}$ static square was presented before an expansion-first condition was initiated and a 23.3$\mathrm{cm}$ static square was presented before a contraction-first condition was initiated. Since the static stimulus preceding the presentation of a particular dynamic condition was of the same physical size and at the same physical distance as the starting size of the particular dynamic condition, the static stimulus was not expected to modify the perceived size or perceived distance of the starting stimulus in either dynamic condition.

\section{Procedure}

Dynamic conditions. Three tasks were completed, using the open-loop procedure, with each of the dynamic conditions. One was to adjust the lateral separation of the unseen posts when the posts were located physically at a near $(\mathrm{N}, 15.7 \mathrm{~cm})$, middle $(\mathrm{M}, 27.9 \mathrm{~cm})$, or far $(\mathrm{F}, 40.0 \mathrm{~cm})$ distance from the observer's eye, in order to in- 
dicate the perceived linear size (width) of the stimulus (see Figure (A). This was completed when the continuous optical change alternately was at its physically smallest stimulus value (perceptually largest distance) and also at its physically largest stimulus (perceptually smallest distance). Since the stimulus remained at each of its terminal sizes for only $1 \mathrm{sec}$ before reversing the direction of its size change, the observers had to view a number of repetitive cycles of the optical change in order to complete a lateral adjustment of the separation of the posts at each of the two terminal positions of the stimulus. The cycles of optical change were continued until the observer was satisfied with the adjustment.

Another task involving the dynamic conditions was to measure the perceived difference in the direction to the left and right side of the stimulus square (the perceived visual angle) at each of the terminal positions of the optical change. In measuring the perceived directions to the stimulus at either the near or far perceived distance of the expansion or contraction, the observer was instructed to turn the head until his/her nose pointed alternately at the left and right edge of the stimulus. The observer was also instructed for each turned position of the head to adjust the unseen posts and hands positioned at the near, middle, or far post distance until the knob at the top of each post felt to be directly under an imaginary straight line extending from the left or right rotated nose position to the left or right edge, respectively, of the stimulus square, using as many cycles of optical size change as needed to complete the task. Figure 1B illustrates the general procedure but without illustrating the pointing of the nose. A somewhat similar procedure for measuring perceived direction has been used by Ono and Nakamizo (1977). In one condition, with the head stationary, the observer fixated and pointed to a small light with an unseen hand, using the bridge of the nose as the origin for the pointing response.

A third task involving the dynamic conditions was the measurement of the sagittal depth perceived between the terminal stimuli in the optical change. This was accomplished by the observer kinesthetically adjusting the depth separation between the inner surfaces of the physically stationary and sagittally movable posts without seeing either the posts or the hands until this sagittal separation was judged to be the same as the depth movement perceived in the cycles of optical change. Again, the cycles of optical change were repeated until the observer was satisfied with the sagittal adjustment.

Static conditions. The static squares were physically identical to the smallest $(7.0 \mathrm{~cm})$ and largest $(23.3 \mathrm{~cm})$ squares used in the terminal sizes of the dynamic conditions. Two tasks using the unseen posts and hands were used with the static presentations. One was the kinesthetic measurement of the perceived linear size of the static square using the near, middle, and far distance positions of the pair of posts (see Figure 1A). The other was the kinesthetic measurement of the perceived direction to the right and left edges of the static stimulus, also using the near, middle, and far distance positions of the pair of posts (see Figure 1B). The latter task again was accomplished by turning the nose to point alternately at a left and right edge of the square and adjusting the separation of the posts to be along the direction of an imaginary line between these positions of the nose and the right or left edge of the square.

Prior to the experimental observations, the differences between the perceived linear and perceived directional tasks were illustrated to the observer by miniature, simplified models depicting the position of the observer, the stimulus, and the lateral and sagittal adjustable posts. Strings on the model were used to show how the lateral separation of the knobs on the top of the posts might be set when the pair of posts were located at different distances from the observer. Parallel strings from the ends of the stimulus to the observer illustrated the post separations for the perceived linear size instructions, and strings converging from the left and right ends of the stimulus to the position of the observer illustrated the post separations for the perceived direction instructions.
Orders of presentation. The order of completing the tasks and the order of presenting the distances of the posts for the linear or angular size adjustments were counterbalanced across observers. The experiment with each observer consisted of two blocks of 22 judgments each, with a 5 -min rest between the blocks. Each block started with the presentation of a stationary (static) square. The order in which the size of the static square (large or small) was presented was counterbalanced across observers. The judgments of perceived size and perceived difference in direction to the two ends of the stimulus in each block, for both the static and dynamic presentations, were made at each of the three physical distances of the posts. If the static square was small, the dynamic presentation that followed was an optical expansion. If the static square was large, the dynamic presentation that followed was an optical contraction. The judgments of perceived depth $\left(d^{\prime}\right)$ were made twice during each dynamic condition, with eight measures obtained from each observer. For each dynamic presentation, the judgments of perceived size and perceived difference in direction to the right and left edges of the stimulus (perceived visual angle) occurred only for the two terminal sizes of the optical change (and not for any intermediate sizes). The order in which the small or large terminal size of an optical expansion or contraction was measured for perceived lateral size or for perceived visual angle was counterbalanced across observers. All of the required judgments for a static or dynamic condition were made at one physical distance of the laterally adjustable posts before making the same kind of judgments with the pair of posts at a different physical distance with the order of post distances counterbalanced between observers.

\section{Results}

In all the graphs of the results of this study, the filled circles represent obtained (perceived) data, the open circles represent physical information, and the filled rectangles represent data computed from a combination of perceived data and physical information.

\section{Obtained and Predicted Perceptions of Motion in Depth}

The average perceptions of motion in depth $\left(d^{\prime}\right)$, in centimeters, obtained from the observer's adjustments of the sagittal separations of the posts in Experiment 1 are shown by the upper filled circles in the left half of Figure 2. These results are from the dynamic conditions in which the first half-cycle of optical change was either an expansion or a contraction. Although the difference between the $d^{\prime}$ obtained from the contraction-first and expansion-first conditions is not large $(24.8$ and $23.2 \mathrm{~cm}$, respectively), this difference is statistically significant $[t(11)=2.87, p=.02]$.

The mean values of perceived depth, $d^{\prime}$, as computed from the obtained values of $S_{\mathrm{s}}^{\prime}$ and $S_{\mathrm{L}}^{\prime}$ using Equation 2 and the physical visual angles of the stimuli $\left(\theta_{\mathrm{s}}=10.0^{\circ}\right.$ and $\theta_{\mathrm{L}}=32.5^{\circ}$ ) are shown by the filled triangles in the left column of Figure 2. Thus, the SDIH as expressed by Equation 2 predicted only about $50 \%$ of the perceived motion in depth obtained from the expansion-first condition and only about $40 \%$ of the perceived motion in depth obtained from the contraction-first condition in Experiment 1 . The predictions from the SDIH as expressed by Equation 2 clearly underestimated the robust perception of motion in depth obtained in the dynamic conditions. 

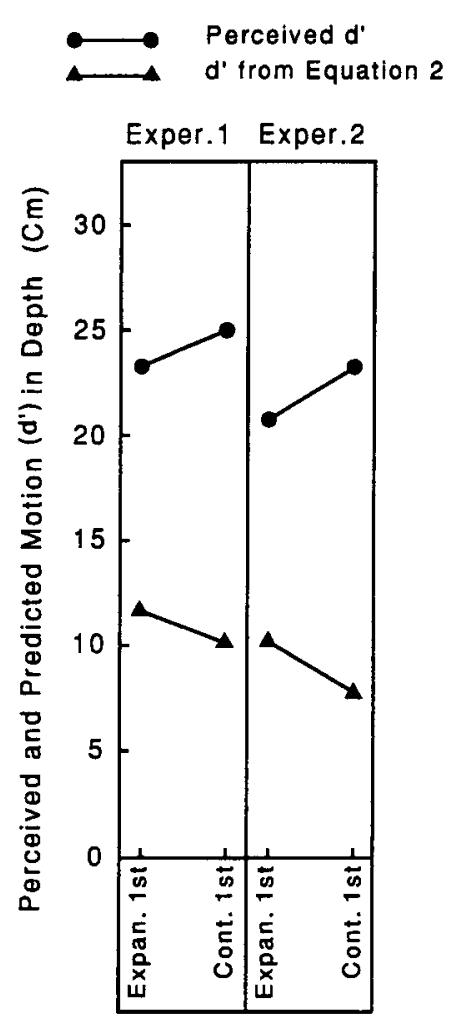

Figure 2. Perceived $d^{\prime}$ and $d^{\prime}$ calculated from Equation 2. The upper, filled circles indicate the average perceived sagittal motion $\left(d^{\prime}\right)$ from the expansion-first and contraction-first conditions as measured directly by using the open-loop kinesthetic, sagittal separations of the pair of posts to duplicate the perceived sagittal motion of the stimulus. The lower, filled triangles indicate the average predicted perception of sagittal motion $\left(d^{\prime}\right)$ from the size-distance invariance hypothesis as obtained by substituting in Equation 2 the measured perceived linear sizes of the stimuli and their physical visual angles.

\section{Perceptions of Linear Size}

Mean results from the perception of linear size, $S^{\prime}$, in centimeters, from Experiment 1 are shown in Figure 3A for the dynamic conditions and static conditions. The upper set of filled circles indicates the perceived linear width from the largest stimulus, and the lower set of filled circles indicates the perceived linear width from the smallest stimulus as measured at the three post distances $(\mathrm{N}$, $M$, and F ). The upper and lower sets of open circles represent the physical linear width of the largest and smallest stimuli, respectively. As indicated, the perceived linear widths of the stimuli generally exceeded the physical terminal sizes presented on the display monitor. This result, in which $S^{\prime}>S$, is expected from the specific distance tendency (Gogel, 1969; Mershon \& Lembo, 1977), which is the tendency for stimuli presented under completely reduced cues of egocentric distance to be perceived at a distance of one or several meters. In the present conditions, only rather weak cues of the egocentric distance of the stimuli on the display monitor (accommodation and accommodative convergence to $40 \mathrm{~cm}$ ) were available. Thus, the effect of the equidistance tendency, although reduced, was not eliminated. Also - of particular importance for this study - it should be noted that, consistent with Figure $1 \mathrm{~A}$, the changes in the physical distance of the laterally adjustable posts $(\mathrm{N}, 15.7, \mathrm{M}, 27.9$, and $\mathrm{F}, 40.0 \mathrm{~cm}$ from the observer) for a constant stimulus size did not produce any consistent change in perceived linear size in the direction expected if measures of linear size had been confused with those of perceived angular size.

The ratio of $S_{\mathrm{s}}^{\prime} / S_{\mathrm{L}}^{\prime}$ is used as a measure of the amount of size constancy present in the different conditions throughout the present study. This concept of the amount of size constancy differs from the usual concept, with the latter referring to the extent to which the perception of size is accurate. Instead, in the present study, the concept refers to the perceived size of the stimulus remaining perceptually constant despite its changing physical size on the monitor. According to this ratio, size constancy is perfect (complete) when $S_{\mathrm{s}}^{\prime} / S_{\mathrm{L}}^{\prime}=1.00$ and is zero when $S_{\mathrm{s}}^{\prime} /$ $S_{\mathrm{L}}^{\prime}=0.30$, with the latter ratio being the ratio of the values of $2 \tan (\theta / 2)$ in Equation 2, which is approximately the ratio $(0.28)$ of the smallest and largest stimuli. The average size constancy obtained from the expansion, contraction, and static conditions was $0.388,0.373$, and 0.339 , respectively. These obtained values are much closer to zero constancy than to perfect constancy. It should be noted, however, that despite the very limited amounts of size constancy present, the perceptions of sagittal motion in the dynamic conditions measured by the sagittal adjustments of the posts and presented in the upper left portion of Figure 2 from Experiment 1 were quite substantial. The magnitude of these perceived motions in depth are inconsistent with the predictions made from Equation 2 of the SDIH. Clearly, a robust perception of motion in depth occurred in the dynamic conditions of Experiment 1 despite the presence of very limited amounts of size constancy.

A three-way analysis of variance (ANOVA) of the $S^{\prime}$ data in the dynamic conditions of Figure 3A was completed using the variables of expansion first or contraction first (M), large or small terminal stimulus (S), and post distance (N, M, or F). Only the perceived size (large or small) was statistically significant at, at least, the .05 level of probability $[F(1,11)=202.18, p<.01]$. A twoway ANOVA also was completed on the $S^{\prime}$ data of Figure $3 \mathrm{~A}$ from the static conditions using the variables of large or small stimulus and post distance $(\mathrm{N}, \mathrm{M}$, or $\mathrm{F})$. Both variables were statistically significant $[F(1,11)=101.53$, $p<.01$ and $F(2,22)=4.14, p=.03$, respectively]. In the dynamic conditions, the perception of linear size $\left(S^{\prime}\right)$ did not differ significantly as a function of the distance of the pair of measurement posts. In the static conditions, the statistically significant change in perceived linear size with post distance, particularly for the largest stimulus, was in the wrong direction to indicate any confusion between perceived linear and perceived angular size.

\section{Perceptions of Angular Size}

Lateral separations of posts for the calculation of perceived visual angle, $\theta^{\prime}$. The results from the lateral separation of the posts to measure the change in perceived 


\section{Results from Experiment 1}

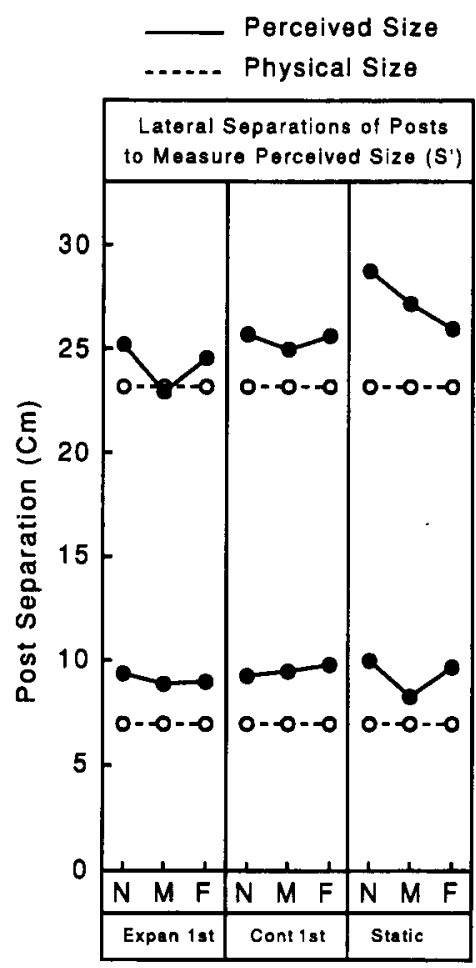

A

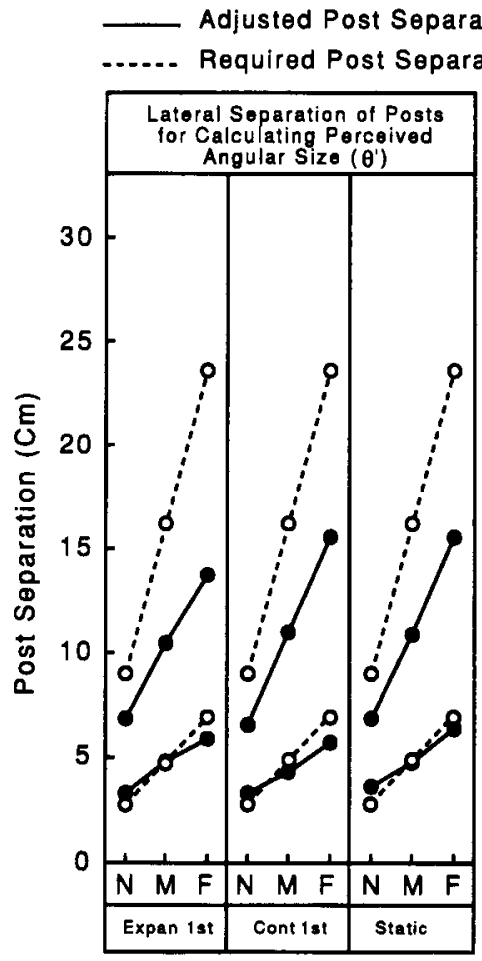

B

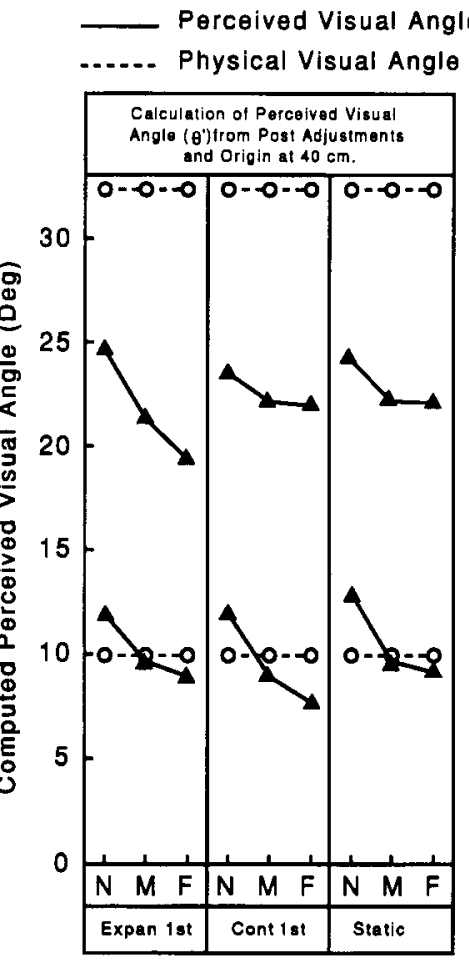

C

Figure 3. Figure $3 \mathrm{~A}$ indicates the average perceived linear sizes $\left(S^{\prime}\right)$ of the stimuli obtained in Experiment 1 by open-loop adjustments of the lateral separations of the pair of posts located at the physical distances of 15.7 (near $[\mathrm{N}]$ ), 27.9 (middle $[\mathrm{M}]$ ), and 40.0 (far [F]) $\mathrm{cm}$ from the observer's eye (with the observer's eye $40 \mathrm{~cm}$ from the stimulus) for the expansion-first, contraction-first, and static conditions. The upper, filled circles represent the data from the largest square stimulus $\left(S_{\mathrm{L}}^{\prime}\right)$ and the lower, filled circles represent the data from the smallest square stimulus $\left(S_{\mathrm{s}}^{\prime}\right)$. The upper and lower sets of open circles in Figure $3 \mathrm{~A}$ are the results that would have been obtained from the largest and smallest stimuli, respectively, if the obtained results had been physically accurate. Figure 3B indicates the open-loop lateral, post separations for calculating perceived angular size $\left(\theta^{\prime}\right)$ from the three physical distances, $15.7(\mathrm{~N}), 27.9(\mathrm{M})$, and $40.0(\mathrm{~F}) \mathrm{cm}$, of the pair of posts from the observer's eye (with the observer's eye $40 \mathrm{~cm}$ from the stimulus) as obtained using the expansion-first, contraction-first, and static conditions of the largest (upper data curves) and smallest (lower data curves) stimuli. The upper and lower sets of open circles in Figure 3B indicate the results that would have been obtained from the largest and smallest stimuli, respectively, if the adjusted separations had been physically accurate. Figure $3 \mathrm{C}$ indicates the calculated perceived visual angles of the largest (upper data curves) and the smallest (lower data curves) stimuli for the three conditions using the results from the three distances of the pair of measurement posts located at the physical distances of $15.7(\mathrm{~N}), 27.9(\mathrm{M})$, and $40.0(\mathrm{~F}) \mathrm{cm}$, from the observer's eye (with the observer's eye $40 \mathrm{~cm}$ from the stimulus). The upper and lower sets of open circles in Figure $3 \mathrm{C}$ indicate the results that would have been obtained from the largest and smallest stimuli, respectively, if the calculations had produced values of $\theta^{\prime}$ that were physically accurate.

direction between the left and right edges of the stimulus in order to calculate perceived visual angle (see Figure 1B) are shown in Figure 3B. The upper set of three curves defined by the filled circles indicate the data from the largest stimulus, $S_{\mathrm{L}}$, and the lower set of three curves defined by the filled circles indicate the data from the smallest stimulus, $S_{\mathrm{s}}$. The upper and lower sets of open circles indicate, respectively, the post separations expected if the separations adjusted by the observer were identical to those required to produce the physical visual angles of the largest and smallest stimuli $\left(32.5^{\circ}\right.$ and $10.0^{\circ}$, respectively). Consistent with Figure 1B, the average post separations provided by the observers increased with each increase in the distance of the posts for both the largest and smallest stimuli and for both the dynamic and static conditions. Comparison of Figure 3B with Figure 3A clearly indicates that the lateral separation of the posts, as a function of the distance of the posts from the observer, consistent with Figure 1, was quite different between perceived angular and perceived linear size. However, changes in the differences between the corresponding filled and open circles in Figure 3B also suggest that the perceived visual angles computed from these data for the same physical visual angle of the stimulus are not likely to be the same. As will be discussed, this is confirmed by Figure 3C.

A three-way ANOVA [expansion first or contraction first (M), largest or smallest stimulus (S), and N, M, or F post distance (D)] was completed on the filled circle data 
of Figure 3B for the dynamic conditions. The variables of largest or smallest stimulus and post distance were statistically significant at, at least, the .05 level of probability $[F(1,11)=198.24, p<.01$ and $F(2,22)=62.07, p=$ .01 , respectively]. In addition, statistically significant interactions at, at least, the .05 level of probability occurred between the variables MD, SD, and MSD.

A two-way ANOVA (stimulus largest $\left[S_{\mathrm{L}}\right]$ or smallest $\left[S_{\mathrm{s}}\right]$ and distance of posts $[\mathrm{N}, \mathrm{M}$, or $\mathrm{F}]$ ) was completed on the filled circle data shown in Figure 3B for the static conditions. Both variables and their interaction were statistically significant $[F(1,11)=167.30, p<.01, F(2,22)=$ $64.92, p<.01$, and $F(2,22)=28.86, p<.01$, respectively].

Calculations of $\theta^{\prime}$ from the dynamic conditions. The filled triangles of Figure $3 \mathrm{C}$ show the values of perceived visual angle in degrees calculated from the filled circle data of Figure 3B, in which the nose was pointed by the observer to each end of the stimuli with the posts at three different distances $(15.7,27.9$, or $40.0 \mathrm{~cm})$ from the observer. Since the head was positioned in a chin cup that permitted head rotation but not lateral displacement of the head, the location of the vertical axis of rotation of the head, and thus presumably the origin of the measured visual angle, was at the chin - that is, approximately at the distance of the eyes. The calculations of the perceived visual angles in degrees used the following information or assumptions: (1) the information as to the left-right post separations at each of the post distances, (2) the origin of the perceived visual angle as being at the distance of the axes around which the head rotated during the nose pointing, (3) the distance of this axis of rotation was sensed by the observer as being $40 \mathrm{~cm}$ from the stimulus, and (4) the felt (kinesthetically perceived) distance of the posts from this origin (physical distances of 15.7,27.7, and $40.0 \mathrm{~cm}$ ) was accurate.

The upper and lower sets of three triangles in Figure 3C are the calculated perceptions $\left(\theta_{\mathrm{L}}^{\prime}\right.$ and $\left.\theta_{\mathrm{s}}^{\prime}\right)$ of the largest, $\theta_{\mathrm{L}}$, and smallest, $\theta_{\mathrm{s}}$, physical visual angles in degrees, respectively. The open circles in Figure $3 \mathrm{C}$ represent the physical visual angles expressed in degrees for the largest and smallest stimuli. In each set of three filled triangles, the perception of the same visual angle was being measured and thus it would be expected that the obtained data curves would be horizontal. It is evident, however, that the computed perceived visual angle was not the same for the same physical visual angle when the posts were at different distances. Instead, for both the largest and smallest stimuli, the value of $\theta^{\prime}$ decreased consistently as the distance of the posts from the observer increased. As will be discussed, it is as though the origin of $\theta^{\prime}$ (the point of rotation of the vertical axis of the head, as perceived by the observer), was at a distance from the stimulus greater than the distance of its physical axis of rotation at the chin.

A second aspect of interest regarding the results shown in Figure 3C is the comparison of the perceived values of $\theta^{\prime}$ as indicated by the sets of filled triangles and the physical size of the visual angles $\theta_{\mathrm{L}}$ and $\theta_{\mathrm{s}}$ as given by the open circles. The values of perceived visual angle of the small- est stimulus averaged over the three distances of the posts for the expansion-first, contraction-first, and static conditions (values of $10.1^{\circ}, 9.6^{\circ}$, and $10.7^{\circ}$, respectively) are similar to the physical visual angle of $10.0^{\circ}$. The corresponding overall average values of perceived visual angle for the largest stimulus, however (values of $21.9^{\circ}, 22.7^{\circ}$, and $22.9^{\circ}$, respectively), are considerably less than the physical value of $32.5^{\circ}$. It seems that two types of error in the measurement of $\theta^{\prime}$ can be distinguished. One is the lack of a constant $\theta^{\prime}$ for the same stimulus measured when the posts were at different distances from the observer. The other is the underestimation of the angular size of the largest stimulus but not of the smallest stimulus.

A three-way ANOVA (expansion or contraction first, largest or smallest stimulus, and post distance) was completed on the $\theta^{\prime}$ data of Figure $3 \mathrm{C}$ from the dynamic conditions. Only the largest versus smallest stimulus and the distance of the posts were statistically significant $[F(1,11)=190.48, p<.01$, and $F(2,22)=8.12, p<.01$, respectively].

Calculations of $\theta^{\prime}$ from the static conditions. The results from the measurements of the change in the perceived direction to the left and right edge of the stimulus, $\theta^{\prime}$, as computed from the static data of Figure 3B also are shown in Figure 3C. The average computed values of $\theta^{\prime}$ in Figure $3 \mathrm{C}$ for the same physical value of $\theta$ increased with each increase in the distance of the posts and were essentially the same as the average $\theta^{\prime}$ values computed from the dynamic conditions. The addition of a continuous optical change, such as occurred in the dynamic conditions, did not seem to modify the measures of perceived visual angle as compared with the results from the static conditions, either in general or as a function of post distance.

A two-way ANOVA (large or small stimulus and post distance) was completed on the static $\theta^{\prime}$ data of Figure $3 C$. Both the large versus small stimulus and the physical distance of the posts were statistically significant $[F(1,11)=$ $166.62, p<.01$, and $F(2,22)=6.10, p<.01$, respectively]

\section{Application of $\theta^{\prime}$ to the Prediction of $d^{\prime}$ From Equation 4}

Using the physical values of $\theta_{\mathrm{s}}$ and $\theta_{\mathrm{L}}$ in Equation 2 resulted in predictions of perceived sagittal motion, $d^{\prime}$, that were in the same direction as, but were considerably smaller than, the perceived sagittal motion as actually measured by the observer's sagittal separation of the posts. It was considered that possibly the predictive ability of the SDIH in the experiment would be increased if perceived rather than physical visual angles were used, as indicated in Equation 4. For this predictive improvement to occur, however, the magnitude of $2 \tan \left(\theta_{s}^{\prime} / 2\right)$ relative to $2 \tan \left(\theta_{\mathrm{L}}^{\prime} / 2\right)$ in Equation 4 must be smaller than that of $2 \tan \left(\theta_{\mathrm{s}} / 2\right)$ relative to $2 \tan \left(\theta_{\mathrm{L}} / 2\right)$ in Equation 2. However, this did not occur. Indeed, Equation 4 makes predictions of the perceived motions in depth opposite to the directions actually obtained. Using the distribution of values of $S_{\mathrm{s}}^{\prime}$ and $S_{\mathrm{L}}^{\prime}$ and of $\theta_{\mathrm{s}}^{\prime}$ and $\theta_{\mathrm{L}}^{\prime}$, the average 
predictions of $d^{\prime}$ from Equation 4 are $-12.8 \mathrm{~cm}$ for the expansion-first and $-9.8 \mathrm{~cm}$ for the contraction-first conditions. Substituting the measures of perceived rather than the physical values of visual angle in Equation 4 worsened the predictions of perceived motion in depth that were obtained using Equation 2.

\section{Discussion}

\section{Relation Between Perceived Linear Size} and Perceived Motion in Depth

According to Experiment 1, a continuous repetitive change in size on the eye, in the absence of any additional stimulus cues to the perception of motion in depth, can produce a robust perception of sagittal motion. On the basis of the literature, this is expected. What is less expected, however, is that this perceived motion in depth occurred despite a quite inadequate amount of size constancy, thus producing a phenomenon inconsistent with the usual form of the SDIH as expressed by Equation 2 . This failure of the appropriate relation to occur between the perceived motion in depth and size constancy in the dynamic conditions is a major finding of Experiment 1 . It supports the general result found by Swanston and Gogel (see Experiments 1 and 3,1986) in which a substantial perceived motion in depth occurred in the expected direction even though a positive amount of size constancy was completely absent. Such results constitute a refutation of the assumption sometimes found implicitly or explicitly in the literature that an optical change, in the absence of any other changing cues of distance, will result in a constant perception of linear size, or more generally will result in the perception of changes in linear size consistent with the changes in perceived motion in depth (see Börjesson \& Von Hofsten, 1972; Hershenson, 1992a, 1992b, 1993; Johansson, 1950, 1964, 1977).

\section{Dependence of Perceived Angular Size on the Perceived Angular Origin}

In addition to the failure of the calculated values of $\theta^{\prime}$ as applied to the SDIH using Equation 4, to predict even the direction of the perceived sagittal motion in the dynamic conditions, a second problem occurs in the interpretation of the $\theta^{\prime}$ data shown in Figure 3C. In both the dynamic and static conditions of Experiment 1, the computed $\theta^{\prime}$ clearly decreased as the distance of the measuring posts from the observer's eyes increased. Since this occurred when measuring the visual angle of a constant stimulus size at a constant distance from the observer, it was unexpected. A possible cause of this result could be a difference between the physical origin of the visual angle at the chin and the origin as perceived and used by the observer. This possibility is diagrammed in Figure 4, which represents a stimulus of physical size $(S)$ and, for convenience, of perceived size $\left(S^{\prime}\right)$ located at a distance, F, from the observer. "Perceived Kinesthetic" in Figure 4 indicates the adjusted separations of the pair of unseen posts and hands (open-loop adjustments) at distances $\mathrm{N}$, $\mathrm{M}$, and $\mathrm{F}$, which are used together with the physical dis-

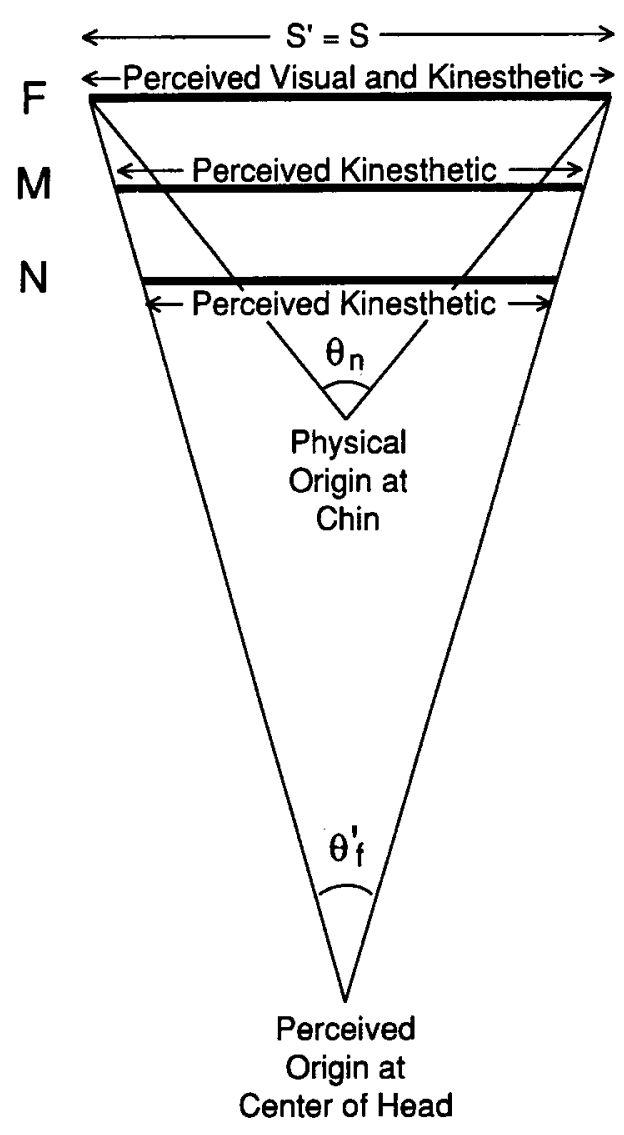

Figure 4. An illustration of the effect of the origin of the perceived visual angle $\left(\theta^{\prime}\right)$ on the calculation of its magnitude, using the lateral separation of the posts at the three distances-near (N), middle (M), or far (F). For simplicity, the illustration represents a situation in which the physical and perceived sizes and the distance of the visual stimulus are identical, but the perceived angular distance of the origin (at the center of the head) is greater than its physical angular distance (at the chin). It should be noted that the greater the distance of the perceived origin, the smaller the measured $\theta^{\prime}$, but the larger the post separations (labeled "Perceived Kinesthetic") for posts at distances less than the perceived distance of the stimulus. The origin used by the observer (the perceived origin) can be identified as that distance at which the calculated value of $\theta^{\prime}$ from the kinesthetic adjustments of the posts is the same at the different post distances. For example, that occurs in Figure 4 only when the origin is at the center of the head, not at the distance of the chin (which also is approximately at the distance of the observer's eyes).

tances of the posts from the origin (pivot) to calculate the perceived visual angle, $\theta^{\prime}$, of the stimulus. If the origin from which $\theta^{\prime}$ is calculated is the origin used by the observer, the values of $\theta^{\prime}$ measured at the different distances of the posts for the same stimulus would all be expected to produce the same $\theta^{\prime}$. In Figure 4, the case is considered in which the physical origin (an origin at the chin around which the head physically pivots) differed from the origin around which the observer perceived the head to be pivoting (an origin at the center of the head). In this case, as illustrated in Figure 4, the origin responsible for producing the post separations is the origin at which, for a 
constant $\theta$, the values of $\theta^{\prime}$ computed from the post separations and post distances from the origin will be constant. In the case illustrated, this is assumed to be the perceived, not the physical, origin when these differ. Thus, the criterion of the elimination of differences in calculated values of $\theta^{\prime}$ obtained from the different post distances can be used to determine the effective distance of the origin of the visual angle of the stimulus when the physical and perceived distance differ.

Figure 5 gives the calculated $\theta^{\prime}$ from Experiment 1 obtained by averaging across the three conditions (expansion first, contraction first, and static) using the near physical origin at the chin $(40 \mathrm{~cm}$ from the stimulus) as compared with a far perceived origin located at the center of the head $(50 \mathrm{~cm}$ from the stimulus). The center of the head, located about $10 \mathrm{~cm}$ behind the eyes (Richards, 1969), was chosen as the likely perceived origin since this is near the distance at which the head rotates when the head is normally turned to point the nose at a stimulus. Clearly, the results from the $\mathrm{N}, \mathrm{M}$, and $\mathrm{F}$ distances of the posts as shown in Figure 5 provide more consistent measures of $\theta^{\prime}$ (for a constant physical $\theta$ of the stimulus) when 50 rather than $40 \mathrm{~cm}$ was used as the distance of the origin from the stimulus. It should be noted, however, as indicated earlier, that although there was no indication that the measures of perceived linear size $\left(S^{\prime}\right)$ were confused by the observer with measures of perceived visual angle $\left(\theta^{\prime}\right)$ of the same stimulus, some intrusion of perceived linear size on the measurement of perceived visual angle is a hypothesis, alternative to the misperception of the angular origin represented in Figure 4. Additional support for the hypothesis of a perceptual error in the position of the origin of rotation, as indicated by the far origin of Figure 4, is discussed in relation to the results from Experiment 3.

\section{EXPERIMENT 2}

It has been suggested (see Hershenson, 1992a, 1992b; Johansson, 1964; Noguchi \& Taya, 1981) that the perceptions obtained from 1- and from 2-D optical changes can differ significantly. Presumably, the differences would be in the direction of perceiving less constancy of object size and a decrease in the magnitude of the perceived motion in depth when 1-D rather than 2-D stimuli are used. This possibility is examined in Experiment 2.

\footnotetext{
Method

\section{Apparatus and Procedure}

The visual alley, apparatus, procedures, orders of conditions, tasks, and observers in Experiment 2 were the same as those used in Experiment 1, with one exception. Instead of the 2-D stimuli (outline squares) used in Experiment 1, the stimuli in Experiment 2 were 1-D (a luminous horizontal line $1.5 \mathrm{~mm}$ in thickness) located in a frontal plane on the display monitor at the height of the observer's right eye, again always at the constant physical distance of $40 \mathrm{~cm}$ from the observer. The optical changes produced the same stimulus lateral widths $(23.3$ and $7.0 \mathrm{~cm})$ and continuous changes
}

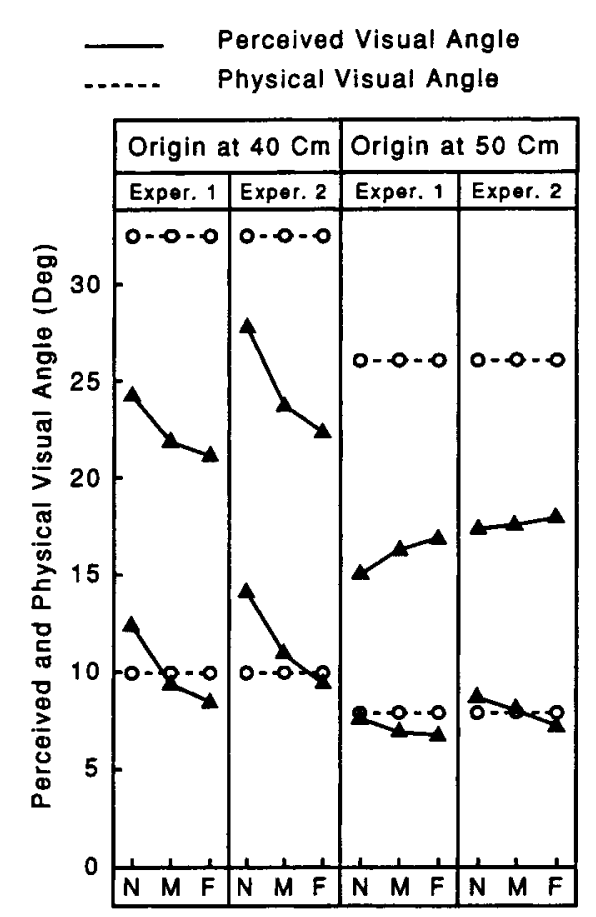

Figure 5. Calculations from Experiments 1 and 2 using the post separations at the three distances-near $(N)$, middle $(M)$, or far (F)-from the observer to measure $\theta^{\prime}$ for the physically largest $\left(S_{\mathrm{L}}\right)$ and smallest $\left(S_{\mathrm{s}}\right)$ stimuli. The perceived origin of the angular measure is assumed to be either at the chin (at its physical origin $40 \mathrm{~cm}$ from the stimulus) or at the center of the head $(50 \mathrm{~cm}$ from the stimulus). The filled triangles represent data calculated from the post separations averaged over the three conditions (expansion first, contraction first, and static) for the near, middle, and far distances of the posts. At the 40-cm physical origin of the angular measure from the stimulus, the distances of the posts from this origin, used in the calculations of the perceived visual angle, were $15.7,27.9$, and $40.0 \mathrm{~cm}$. At the hypothetical, perceived location of the angular origin at the center of the head $(50 \mathrm{~cm}$ from the stimulus), the distances of the posts from this origin used in the calculations of the perceived visual angles were taken to be $25.7,37.9$, and $50.0 \mathrm{~cm}$ from this origin. Clearly in Experiment 1 (and also in Experiment 2), the data curves (the filled triangles) were more constant as a function of post distance for both the upper curves (the largest stimulus) and the lower curves (the smallest stimulus) for a perceived origin at $50 \mathrm{~cm}$ as compared with the physical (actual) location of the origin at $40 \mathrm{~cm}$. The effective (perceived) origin around which the head was rotated in pointing the nose was approximately at $50 \mathrm{~cm}$ from the stimulus despite the fact that the head rotated physically around a vertical axis at the chin, $40 \mathrm{~cm}$ from the stimulus. Again, the open circles represent the results that would have been obtained from the largest and smallest stimuli if the calculations had produced values of $\theta^{\prime}$ that were consistent with the 40 - and $50-\mathrm{cm}$ distances of the origins from the stimulus.

in width, as in Experiment 1, and, thus, the same simulations of lateral sizes and sagittal motions. Static line stimuli with the same width (right to left physical extent) as that of the starting stimulus of the optical change (expansion first or contraction first) were also presented in Experiment 2 immediately prior to presenting the dynamic condition of the same starting size. Again, the observation was always monocular with the left eye covered. 


\section{Results}

\section{Perceptions of Motion in Depth}

Dynamic conditions. The results from the perception of sagittal motion from the dynamic conditions of Experiment 2 are shown in the upper pair of data points in the right column of Figure 2. As in Experiment 1, the contraction-first condition resulted in a somewhat larger average $d^{\prime}$ than was obtained from the expansion-first condition, with this difference of $2.4 \mathrm{~cm}$ being statistically significant $[t(11)=2.53, p<.03]$. The overall $d^{\prime}$ of $21.9 \mathrm{~cm}$ in Experiment 2 was somewhat less than the $24.0 \mathrm{~cm}$ overall obtained in Experiment 1. A two-way ANOVA of the depth data was completed using the variables Experiment 1 or Experiment 2 and expansion first or contraction first. Only the expansion- or contractionfirst variable was statistically significant $[F(1,11)=10.09$, $p<.01]$.

\section{Perceptions of Linear Size}

Dynamic conditions. The results from the perception of linear size $\left(S^{\prime}\right)$ from Experiment 2 are shown in Figure $6 \mathrm{~A}$. The structure and meaning of the data presented in Figure 6 parallel those of Figure 3, with the upper and lower average data points referring to the largest and smallest stimuli, respectively, the solid and open data points (circles) referring to the obtained (perceptual) and physical data, respectively, and the filled triangles referring to the combined use of physical and perceptual data. A three-way ANOVA of the $S^{\prime}$ data was completed using the variables of expansion or contraction first (M), dynamic large or small stimuli (S), and post distances (D). Only the dynamic large or small stimulus and its interaction with post distance was statistically significant at, at least, the .05 level of probability $[F(1,11)=197.0, p<$ .01 , and $F(2,22)=3.46, p<.05$, respectively].

The average results from the perception of linear size $\left(S^{\prime}\right)$ for the dynamic conditions of Experiment 2 are somewhat larger than those obtained from Experiment 1 (compare Figures 3A and 6A). The amount of size constancy in the two experiments was again calculated by the ratio $S_{\mathrm{s}}^{\prime} / S_{\mathrm{L}}^{\prime}$, where ratios of 1.000 and 0.300 represent perfect and zero constancy, respectively. In Experiment 1, the average values of these ratios were .388 and .373 for the expansion-first and contraction-first conditions. In Ex-

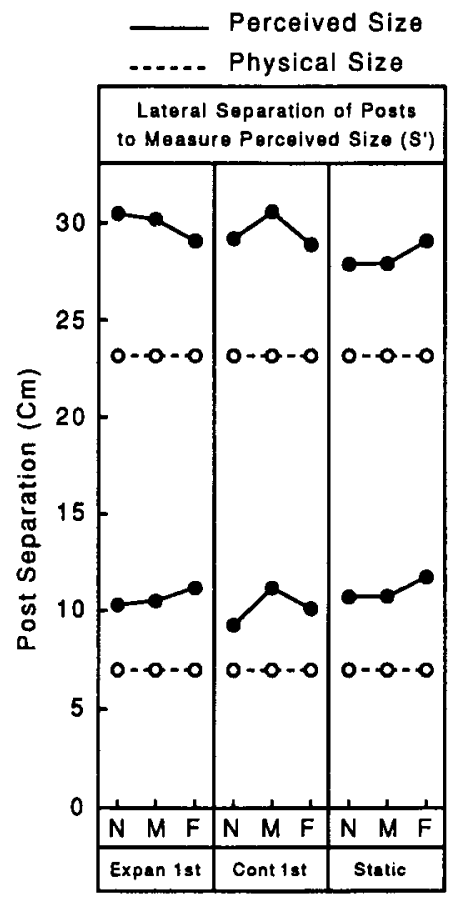

A

\section{Results from Experiment 2}

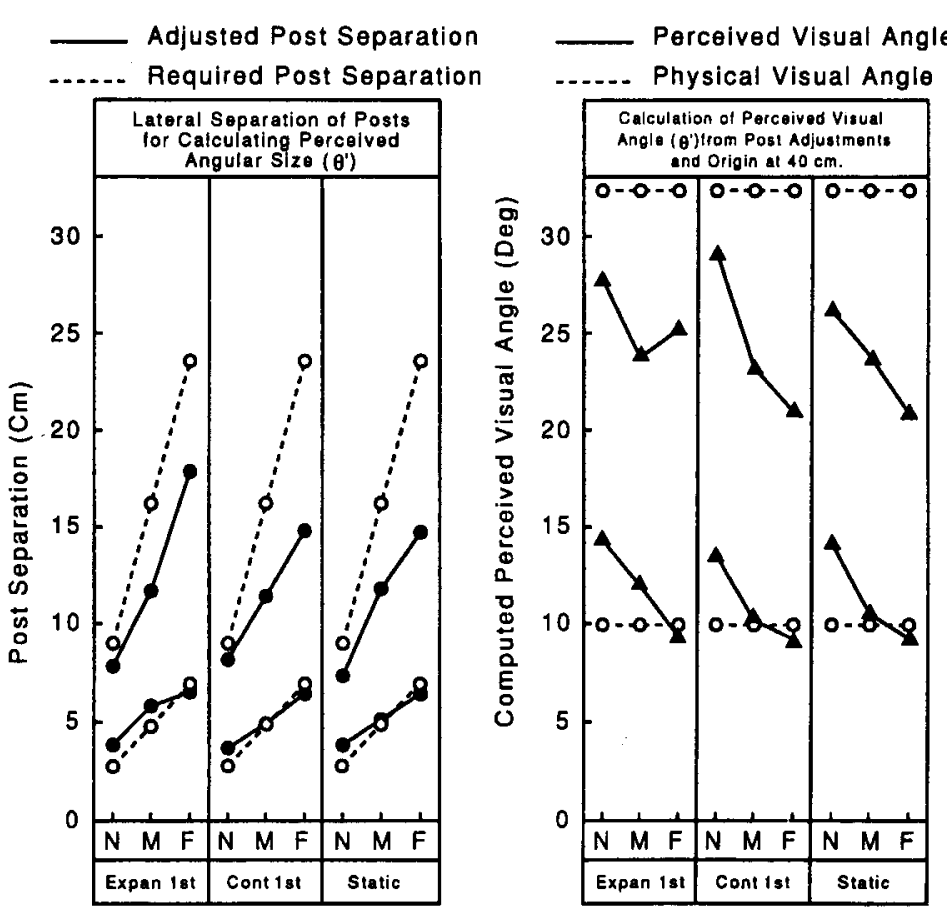

Figure 6. This figure, from Experiment 2, has the same structure and same interpretation as Figure 3 from Experiment 1. Again, the upper and lower graphs refer, respectively, to the largest and smallest stimuli, with the solid data points indicating perceived characteristics and the open data points indicating physical characteristics. The results (using openloop procedures) indicate that (1) the perceived linear size $\left(S^{\prime}\right)$ was essentially independent of the distance of the measuring posts (Figure 6A), (2) the results from the post separations used to measure perceived angular size increased as the distance of the measuring posts from the physical origin at $40 \mathrm{~cm}$ from the stimulus (at the chin) increased (Figure $6 \mathrm{~B}$ ), and (3) using the same (either largest or smallest) stimulus, the perceived angular size computed from the post adjustments decreased as the distance of the posts from the physical origin at $40 \mathrm{~cm}$ from the stimulus (at the chin) increased (Figure 6C). N, near; $M$, middle; F, far. 
periment 2 , they were .347 for both of these conditions. These amounts of size constancy were small in both experiments, with the size constancy from Experiment 1 somewhat larger than that from Experiment 2.

A four-way ANOVA of the $S^{\prime}$ data from the dynamic conditions as obtained from Experiments 1 and 2 used the variables $M, S$, and D plus the additional variable of Experiment 1 or 2 (E). The overall difference in $S^{\prime}$ from Experiments 1 and 2 (a difference of about $3 \mathrm{~cm}$ averaged over the large and small stimuli) was significantly larger in Experiment 2 than in Experiment $1[F(1,11)=5.37$, $p<.04$ ], as was the variable of large or small stimulus size averaged over the two experiments $[F(1,11)=267.21, p<$ $.01]$. In addition, significant interactions at, at least, the .05 level occurred between the variables ES, ED, and ESD.

Static conditions. The mean results in centimeters for the perception of linear size $\left(S^{\prime}\right)$ in the static conditions of Experiment 2 are shown in the right panel of Figure 6A. The results are similar to the $S^{\prime}$ values from the dynamic conditions except that $S_{\mathrm{L}}^{\prime}$ and $S_{\mathrm{s}}^{\prime}$ from the static conditions are somewhat smaller and larger, respectively. The ratios of the smallest $\left(S_{\mathrm{s}}^{\prime}\right)$ and largest $\left(S_{\mathrm{L}}^{\prime}\right)$ perceived linear sizes from the static conditions were .339 and .391 for Experiments 1 and 2, respectively. A two-way ANOVA of the $S^{\prime}$ data of Experiment 2 (stimulus large or small and post distances $\mathrm{N}, \mathrm{M}$, or F) was completed. Only the large or small size of the stimulus was statistically significant $[F(1,11)=158.96, p<.01]$. A three-way ANOVA was completed comparing the static results in Experiments 1 and 2 using the variables of Experiment 1 or 2, stimulus large or small, and post distance $\mathrm{N}, \mathrm{M}$, or $\mathrm{F}$. Only the stimulus large or small $[F(1,11)=158.31, p<$ $.01]$ and the interaction of Experiment 1 or 2 and post distance $[F(2,22)=3.98, p<.03]$ were statistically significant at, at least, the .05 level of probability.

\section{Perception of Angular Size}

Dynamic conditions. The results from the lateral separation of the posts to indicate the change in perceived direction between the left and right edges of the stimulus from Experiment 2 are shown in Figure 6B. As in Experiment 1 , the filled circle data of Figure $6 \mathrm{~B}$ are the average lateral separations of the pair of posts needed to align the posts under imaginary lines extending between the pointing nose and the left and right edges of the stimulus when the posts were at the near, middle, and far distances from the observer. A comparison of these results and the $S^{\prime}$ data from Figure 6A shows that, unlike the adjusted separation when measuring perceived linear size, the adjusted separation when measuring perceived angular size increased regularly with each increase in post distance. A three-way ANOVA (expansion or contraction first [M], large or small sized terminal stimuli [S], and post distance [D]) was applied to the data of Figure $6 \mathrm{~B}$ from Experiment 2. The variables of stimulus size and post distance were statistically significant at, at least, the .05 level $[F(1,11)=140.06, p<.01$, and $F(2,22)=63.77$, $p<.01$, respectively], as were the interactions of SM, $\mathrm{SD}$, and SMD.
As in Experiment 1, in Experiment 2 the results in Figure $6 \mathrm{C}$ were obtained from distributions of values of $\theta^{\prime}$ computed for each observer from perceptions of the directional differences to the right and left ends of the stimulus as measured by the post separations given in Figure 6B. Also, as in Experiment 1, for the purpose of calculating the perceived visual angles in Experiment 2, the origin was assumed to be perceptually as well as physically at the distance of the chin cup $(40 \mathrm{~cm}$ from the stimulus). Thus, the values of $\theta^{\prime}$ were computed from the lateral adjustments of the posts (in Figure 6B) using the $40-\mathrm{cm}$ origin and the three physical distances of the posts from the origin (assuming that these kinesthetically perceived and physical post distances of $15.7,27.9$, or $40.0 \mathrm{~cm}$ were identical). A three-way ANOVA (expansion or contraction first, large or small stimulus, and post distance) was completed for the $\theta^{\prime}$ averaged in the dynamic conditions of Figure $6 \mathrm{C}$. Only the variables of large or small size and distance of posts were statistically significant at, at least, the .05 level $[F(1,11)=122.39, p<.01$, and $F(2,22)=8.68, p<.01$, respectively]

Static conditions. The $\theta^{\prime}$ values from the static condition shown in the right panel of Figure $6 \mathrm{C}$ were computed from the static condition data of Figure $6 \mathrm{~B}$, again assuming an origin of $40 \mathrm{~cm}$ from the stimulus. A two-way ANOVA, using the variables from the static conditions of large or small stimulus and post distance, $\mathrm{N}, \mathrm{M}$, or F, was completed. Both the variables of stimulus size and post distance were statistically significant at, at least, the .05 level $[F(1,11)=132.92, p<.01$, and $F(2,22)=8.17, p<$ .01 , respectively]. As noted in Experiment 1, in Experiment 2 for all three conditions (expansion first, contraction first, or static), the calculated $\theta^{\prime}$ decreased for a constant stimulus size as the distance of the measuring posts from the observer increased. Furthermore, the patterns of values of $\theta^{\prime}$ calculated in the three conditions and two experiments for a particular physical angle were generally similar. Such results suggest that whatever the factors that were determining the values of $\theta^{\prime}$, they were similar in all conditions and in both experiments.

\section{Application of $\theta^{\prime}$ to the Prediction of $d^{\prime}$ From Equation 4}

In Experiment 2, as in Experiment 1, Equation 4, unlike Equation 2, was unable to predict even the direction of the $d^{\prime}$ obtained using the average values of $S_{\mathrm{s}}^{\prime}, S_{\mathrm{L}}^{\prime}$, and $\theta_{\mathrm{s}}^{\prime}, \theta_{\mathrm{L}}^{\prime}$ in the dynamic conditions. In Experiment 2, the predicted $d^{\prime}$ from Equation 4 was $-14.4 \mathrm{~cm}$ for the expansion-first condition and $-15.4 \mathrm{~cm}$ for the contraction-first condition. Again, such errors in predicting the direction of the perceived motion, as is indicated by the predicted minus values of $d^{\prime}$, must reflect substantial errors in the measurement of $\theta^{\prime}$.

A four-way ANOVA compared the $\theta^{\prime}$ data of Experiments 1 and 2 for the factors Experiment 1 or 2, expansion or contraction first, large or small terminal stimuli, and post distance. Only the large or small terminal stimulus, the interaction of this factor and post distance, and the four-factor interaction were statistically significant 
beyond the .05 level of probability $[F(1,11)=334.48, p<$ $.01 ; F(2,22)=4.48, p<.02$; and $F(2,22)=4.69, p<.02$, respectively].

\section{Discussion}

Statistically significant differences are present between Experiments 1 and 2 for the perception of size, the magnitude of perceived sagittal motion, and the measurement of $\theta^{\prime}$. However, none of these differences are sufficient to suggest that the process underlying the two experiments are basically different. Both experiments demonstrate that in situations in which cues of changing distance, other than changing size on the eye, are absent (as in an optical expansion or contraction viewed under otherwise reduced conditions), the perception of motion in depth can be robust even though only small (inadequate) amounts of size constancy are available.

Several equations other than Equations 2 or 4 have been considered as representing the SDIH (see Higashiyama \& Shimono, 1994). In the present study, for example, if the basic equation representing the SDIH were taken as

$$
S^{\prime} / D^{\prime}=K[2 \tan (\theta / 2)],
$$

where $K$ is approximately 2.3 , it would provide reasonably accurate predictions of the obtained values of $d^{\prime}$. Or, more generally, expressing the SDIH as a power function, that is,

$$
S^{\prime} / D^{\prime}=K \theta^{n},
$$

where $\theta$ or $\theta^{\prime}$ is in radians and $K$ and $n$ are situational constants (Foley, 1967, 1968; Gogel, 1971; Oyama, 1974) would very likely considerably improve the predictions of obtained $d^{\prime}$ from those provided by Equations 2 or 4 . But, there is a rationale for using Equations 1 and 3 to be tested as representing the SDIH and applying these as Equations 2 and 4 to the $d^{\prime}$ data of Experiments 1 and 2 . The rationale is that the visual system, if it is to produce, under the proper conditions, veridical perceptions of size and distance, must, under those conditions, be capable of producing a ratio of perceptions that can model physical events. But, a ratio of perceived size, $S^{\prime}$, and perceived distance, $D^{\prime}$, can model the physical world only when $S^{\prime} / D^{\prime}=S / D$. In this case, since $S / D=\theta$ in radians (approximately), $S^{\prime} / D^{\prime}$ also must equal $\theta$ in radians-that is, $S^{\prime} / D^{\prime}=\theta$ in radians $=2 \tan (\theta / 2$ in degrees $)$, which is Equation 1 . Thus, $S^{\prime} / D^{\prime}$ in perceived space models physical space only when $K$ in Equations 5 and 6 and $n$ in Equation 6 are unity. Conversely, if neither $K$ nor $n$ are unity, the ratio of perceived size and perceived distance cannot model physical events, and veridical perceptions of this ratio cannot be identified by Equations 5 or 6 . Under the assumption that such veridical perceived ratios are attainable, it seemed appropriate to use the predictive ability of Equations 2 or 4 in the present study.

Although the axis of the head rotation was physically at the chin (origin at $40 \mathrm{~cm}$ ), the calculations of $\theta^{\prime}$ from the observer's adjustments of the posts suggests that the perceived axis of rotation in both Experiments 1 and 2 was beyond the chin, perhaps near the center of the head (origin at $50 \mathrm{~cm}$ ). As is shown in Figure 5, this is supported by the data from these experiments, since in both experiments the calculation of $\theta^{\prime}$ with the origin at the center of the head (origin at $50 \mathrm{~cm}$ from the stimulus) provided a considerably more consistent measure of $\theta^{\prime}$ from the same stimulus than for an origin at the chin (origin at $40 \mathrm{~cm}$ from the stimulus). This interpretation-that the observer perceived the origin for the angular size of the stimulus to be beyond the chin (or eyes) in the direction of the center of the head rather than located at the physical distance of the chin-is examined further in Experiment 3.

\section{EXPERIMENT 3}

As shown in Figures $3 \mathrm{C}$ and $6 \mathrm{C}$, the expectation that the values of perceived angular size-computed from the post separation at the different distances of the pair of posts from the observer, with the physical distance of the pivot (origin) at the chin-would be essentially equal was not realized. Two hypotheses were proposed to explain this unexpected result. One, as illustrated in Figure 4, is that the observer misperceived the position of the physical axis of head rotation (the physical origin) from which $\theta^{\prime}$ actually was obtained. Specifically, probably because of past experience when turning the head, the axis (origin) of rotation was assumed to be nearer the center of the head rather than at the distance of the eyes. Consistent with Figure 5 , differences in $\theta^{\prime}$ as a function of the physical distance of the measuring posts were clearly reduced in both Experiments 1 and 2 when $\theta^{\prime}$ was computed from the perceived origin at the center of the head $(50 \mathrm{~cm}$ from the stimulus) rather than from the physical origin (pivot) at the chin (40 cm from the stimulus). This "error in origin" hypothesis is further examined in Experiment 3, using only static stimuli and measuring only the perception of angular size.

\section{Method}

\section{Observers}

The observers were 16 undergraduate students ( 6 women and 10 men) from an introductory course in psychology. All indicated that they required no optical correction in the right eye, and all were naive as to the hypotheses of the experiment.

\section{Apparatus}

The stimuli. The stimuli consisted of two luminous static white outline squares 23.3 or $7.0 \mathrm{~cm}$ wide that were presented one at a time with a $4.0-\mathrm{mm}$ circular dot in the center of each square. The sides of the square were lines $2.0 \mathrm{~mm}$ wide for the large square and $1.5 \mathrm{~mm}$ wide for the small square. Unlike the stimuli used in the previous two experiments, the squares were made by placing a cardboard mask over an electroluminescent panel. Mounting grooves at the front of the panel permitted the experimenter to present either the small or the large square at a constant distance of $40 \mathrm{~cm}$ from the observer. The center of each stimulus was approximately at the observer's eye level and directly forward of the observer's nose when the head was pointing straight ahead. The luminance of the stimuli was $0.8 \mathrm{log}$ units above foveal threshold for 20 -min dark adaptation of a middle-aged eye. The observation throughout the experiment, as in the two previous experiments, was with the right 
eye only. The left eye was covered by an eye patch. Unlike the previous two experiments, optical expansion and contraction were not used. The stimuli in Experiment 3 were always static; that is, they did not change in size while being viewed by the observers.

Visual alley and measuring posts. The stimulus, presented in an otherwise completely dark surround, was viewed through an unseen aperture $40.0 \mathrm{~cm}$ wide and $10.2 \mathrm{~cm}$ high that could be occluded or opened by a sliding shutter. As in Experiments 1 and 2, a hand-adjustment apparatus for the measurement of visual angle was located in front of the observer, approximately at waist level. This hand adjustment apparatus was used only for the measurement of perceived visual angle $\left(\theta^{\prime}\right)$ since, unlike Experiments 1 and 2, perceived linear size $\left(S^{\prime}\right)$ was not measured in Experiment 3 . The adjustment apparatus consisted of a pair of vertical metal posts, $1.25 \mathrm{~cm}$ on a side, which moved along a track oriented in the observer's frontoparallel plane with the track either 15.7 or $40.0 \mathrm{~cm}$ from the observer's eye. Each post of the pair was $9.0 \mathrm{~cm}$ in length and had a small knob, $3 \mathrm{~mm}$ wide, at the top with the top $23.0 \mathrm{~cm}$ below the center of the stimulus (below eye level). The left and right posts, when moved to touch together, were centered on the observer's midline, and neither could be moved past the center position. On half of the trials the track and the posts that moved laterally on the track were positioned $15.7 \mathrm{~cm}$ from the observer's eye, and on the other trials at $40.0 \mathrm{~cm}$ from the observer's eye. The observer was able to independently move each post of the pair horizontally left or right along the track with one post in each hand with the thumbs resting on the knobs. Each post was separately connected to a rack-andpinion mechanism that rotated a potentiometer as the post was moved. The potentiometers were interfaced to a computer's A/D input in order to record the observer's adjustment of the posts. The maximum amount that the left and right posts could be separated laterally was $64.0 \mathrm{~cm}$. Nothing - the posts, the observer's hands used in adjusting the posts, or any object other than the stimuluswas visible to the observer during the post adjustments.

The head restraining mechanism. The chinrest that had been used in Experiments 1 and 2 to specify the pivot around which the head rotated was not present in Experiment 3. Instead, the vertical axis around which the head rotated (called the "pivot position" or "physical origin of the angle") was determined by a set of snugly fitting, padded ear cups connected by a rigid metal band that passed over the top of the head. The top of this metal band was attached to a structure that specified the point around which the head rotated as the head was turned. This vertical rotation axis of the head (pivot distance or angular origin) was either at the distance of the eyes ( $40 \mathrm{~cm}$ from the stimulus) or $10 \mathrm{~cm}$ behind the eyes $(50 \mathrm{~cm}$ from the stimulus) at approximately the center of the head. A drawing of this apparatus, simplified to illustrate the principle of its operation, for producing the 40- and 50-cm physical origins of the visual angles of the stimuli, is shown in Figure 7. The actual (physical) construction of this apparatus was more elaborate than that indicated in Figure 7 in order to meet the requirement of physical stability while maintaining the eyes of the observer always at $40 \mathrm{~cm}$ from the stimulus.

\section{Procedure}

Instructions. The observer was given preliminary instructions using a model similar to that used in the preliminary instructions of Experiments 1 and 2 in order to explain the apparatus for adjusting the lateral position of each post so that the knob on the top of a post was felt to be located directly below an imaginary line connecting the base of the nose and the left or right edge of the square stimulus.

Two conditions were used in the measurement of the change in the perceived direction to the right and left edges of the stimulus (the perceived visual angle, $\theta^{\prime}$ ). One, as in Experiments 1 and 2, involved pointing the nose to the direction of the right or left edge of the stimulus and adjusting the right and left posts, using the openloop procedure, to be beneath the imaginary lines extending from the base of the nose to the right or left edge of the stimulus. In the other condition, the observer was again instructed to adjust the posts to be beneath imaginary lines extending from the base of the nose to the right and left edges of the stimulus. But, in this condition the head restraint was pinned in place to prevent any head rotation, while keeping the nose pointing always at the center of the stimulus. When the condition involved head rotation, the observer was instructed to rotate the head slowly and smoothly to avoid any slipping of the ear cups on the head. In all conditions, the observer was told to look back and forth between the left and right edges of the stimulus to be certain that the posts were adjusted simultaneously to the perceptual criterion before the amount of the post adjustments was recorded on the computer. From these adjusted post separations using open-loop procedures and the physical distance of the posts from the rotational origin, the measures of perceived visual angles $\left(\theta^{\prime}\right)$ were calculated.

It has been found from measures indicating the perceived positions of and perceived extent between the vertices of the MüllerLyer illusion that these two kinds of perceptions can differ (Gillam

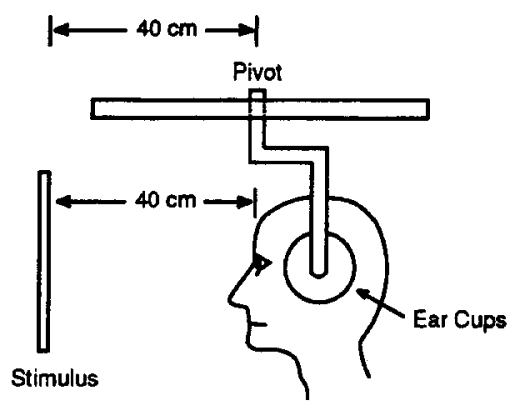

A
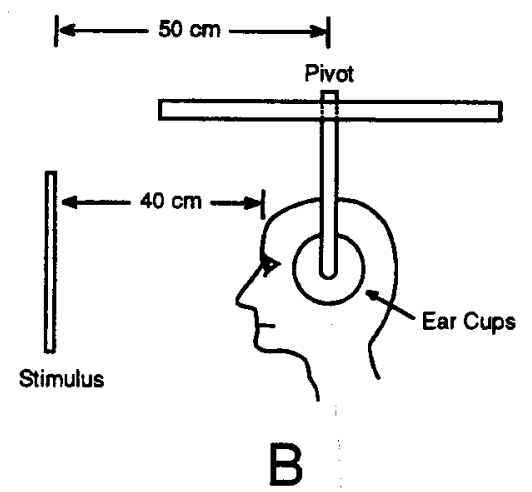

Figure 7. A simplified drawing of the apparatus used in Experiment 3 to change the distance of the origin (the distance of the vertical axis around which the head rotates) when pointing the nose at the left or right edge of the stimulus or keeping the head stationary. In Figure $7 \mathrm{~A}$, the physical origin (pivot) was at $40 \mathrm{~cm}$ from the stimulus (essentially at the distance of the chin, the base of the nose, and the eye). In Figure 7B, the physical origin (pivot) was at $50 \mathrm{~cm}$ from the stimulus - that is, near the center of the head. It should be noted that for either origin, the physical distance of the stimulus was $40 \mathrm{~cm}$ from the observer's eye. For the stationary (no rotation of the head) condition, the head was restrained to point in a straight-ahead position (a no-pivot condition) with the stimulus again at $40 \mathrm{~cm}$ from the observer's eye. 
\& Chambers, 1985; Mack, Heuer, Villardi, \& Chambers, 1985). From this it can be argued that, in the present study, the measures of perceived visual angle $\left(\theta^{\prime}\right)$ obtained by the difference in the perceived directions to the right and left edges of the stimulus did not provide an accurate measure of perceived angular extent. Predebon (1995) conducted a study that yielded results consistent with those from the above studies in three experiments, but in a fourth experiment provided a different interpretation of these results. It was indicated that whether the Müller-Lyer illusion modified perceived position as measured by pointing as well as perceived extent depended on the attentional inspection strategies used by the observers as determined by the procedures. When the observers were not informed as to the vertex to which they were to point until after the illusion had been presented (a postcued condition), the pointing response, like the extent response, reflected the illusory distortion. This suggests that the precued condition had the effect during the pointing task of restricting the attention to the designated vertex, thereby, for that task, selectively reducing or eliminating the illusory effect. But, it seems unlikely that a limitation in examining the entire stimulus would have occurred in Experiments 1 and 2 of the present study, since in those experiments the observers were informed before beginning the task that perceived direction was to be measured with respect to both ends of the stimulus. Nevertheless, in Experiment 3 this aspect of the pointing task was emphasized in the instructions. That perceived visual angle can be sensitive to illusory increments in extent has been shown by Higashiyama (1992) using a horizontal-vertical illusion for the stimulus and an adjustable hand-held protractor for the measures of perceived visual angle.

Implicit in Figure 1 is another factor to be considered when contrasting perceived extent measured by (1) separating the hands to directly duplicate the lateral extent of the stimulus and (2) using the hands to point beneath the left and right ends of the stimulus. If the hands are at the physical but not the perceived distance of the stimulus when making these measures, the two measures will differ. This misperception of the distance of the stimulus from the observer will modify the first measure but is unlikely to modify the second measure. This is because the second but not the first measure is subject to Emmert's law (Emmert, 1881). If the perceived distance is sufficiently greater than the physical distance of the stimulus, the first measure will exceed the second. If the perceived distance is sufficiently less than the stimulus, the second measure will exceed the first. For stimuli fairly close to the observer, especially when presented under reduced conditions of observation, a positive error in the perceived distance is very likely, thereby resulting in a larger measure of perceived extent from the first than from the second measure. Thus, before interpreting results as indicating differences in the visual processing of extent and position, it must be shown that the pointing hands are directly beneath the perceived rather than the physical distance of the stimulus whenever the perceived and physical distances differ.

Stimulus variables. Three variables were present in Experiment 3 . One variable was the physical positions around which the head turned (the physical origins) and from which the perceived directions to the left and right edges of the stimulus were to be judged. In the two conditions in which the head was turned to point the nose in the directions of the stimulus edges, these physical origins were specified by the position of the vertical axis (pivot) around which the head rotated, which was fixed by the apparatus to be at either 40 or $50 \mathrm{~cm}$ from the stimulus. In the remaining condition, the head was stationary. However, in this stationary condition also the 40 - and 50 - $\mathrm{cm}$ distances from the stimulus can be considered as providing alternate physical origins for judging directions and perceived visual angles. The near $(40-\mathrm{cm})$ distance was likely to be the distance position of the cyclopean eye from which direction is often considered to be perceived. The far $(50-\mathrm{cm})$ distance is at the position experienced as being the axis of head rotation that occurred when the head was turned to view stimuli. Thus, the physical origins at 40 or $50 \mathrm{~cm}$ from the stimulus can be expected to provide alternative origins for judging visual angle in all three conditions. However, according to Experiments 1 and 2, the effective origin (the perceived origin), as identified by the criterion of a constant $\theta^{\prime}$ despite differences in post distances, is expected to be closer to $50 \mathrm{~cm}$ than to $40 \mathrm{~cm}$ from the stimulus. A second variable was whether the stimulus was physically the largest stimulus $\left(S_{\mathrm{L}}=23.3 \mathrm{~cm}\right.$ wide $)$ or the smallest stimulus ( $S_{\mathrm{s}}=7.0 \mathrm{~cm}$ wide). A third variable was the physical distance of the pair of posts used in the measurement of $\theta^{\prime}$. In the case in which the physical origin (pivot) was at $40 \mathrm{~cm}$ from the stimulus (at approximately the base of the nose), the pair of posts was either at 15.7 or $40.0 \mathrm{~cm}$ from this physical origin. In the case in which the physical origin was at $50 \mathrm{~cm}$ from the stimulus (at approximately the center of the head), the pair of posts was at either 25.7 or $50.0 \mathrm{~cm}$ from the physical origin. In all conditions, the physical distance of the stimulus was always at $40 \mathrm{~cm}$ from the observer's eyes, as shown in Figure 7.

Orders of presentation. Before beginning the experimental trials, the observer received a single practice trial using the conditions of the experimental trial to be presented first. The stationary head condition occurred first for half the observers and last for the remaining observers. For the two head-rotation conditions, half the observers had the physical origin (pivot) at the base of the nose (the $40-\mathrm{cm}$ condition) first and the physical origin (pivot) at the center of the head (the $50-\mathrm{cm}$ condition) second, with the reverse order for the other half. The posts were adjusted first at the near and then at the far distance for half the observers, with the order reversed for the other half. Half the observers were presented with the small stimulus before the large stimulus, with the order reversed for the remaining half. Half the observers pointed the nose at the left edge of the stimulus prior to pointing at the right edge of the stimulus, with the order reversed for the other half. These conditions were assigned to individual observers in such a way that no 2 observers received the same order. All the conditions within a given head-rotation or head-stationary condition were completed before changing to the next head-rotation or head-stationary condition. Post adjustments at both distances of the posts were completed for one stimulus size before changing to the other stimulus size.

\section{Results}

The average results from Experiment 3 involving the lateral adjustments of the posts and their application in calculating the perceived visual angles of the stimuli are shown in Figure 8. As in Figures 3 and 6, in Figure 8, the lower, filled points represent average results from using the small stimulus and the upper, filled points represent the average results from using the large stimulus. The filled circles in Figure 8A show the observer's adjustments of the lateral separations of the posts at the near $(\mathrm{N})$ and far (F) post positions when the physical (actual) axis of the rotation of the head (the pivot) was at the base of the nose $(40 \mathrm{~cm})$, at the center of the head $(50 \mathrm{~cm})$, or in the stationary head condition, when it was absent. The filled triangles of Figures $8 \mathrm{~B}$ and $8 \mathrm{C}$ represent the perceived visual angles $\left(\theta^{\prime}\right)$ calculated using the post adjustments of Figure $8 \mathrm{~A}$, assuming that the perceived pivot distance from the stimulus was $40 \mathrm{~cm}$ in Figure $8 B$ and $50 \mathrm{~cm}$ in Figure $8 \mathrm{C}$. The $40-\mathrm{cm}$ assumed distance of the origin of the perceived visual angle from the stimulus in Figure 8B and the $50-\mathrm{cm}$ assumed distance of the origin of the perceived visual angle from the stimulus in Figure $8 \mathrm{C}$ were 


\section{Results from Experiment 3}

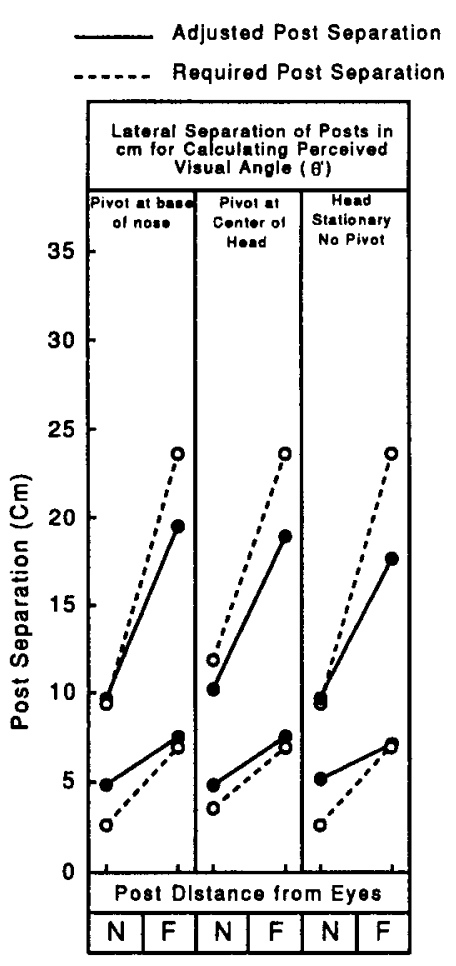

A

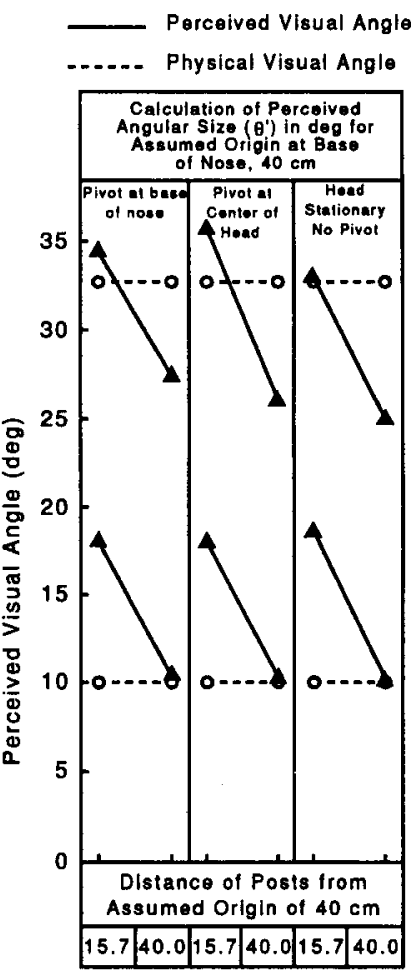

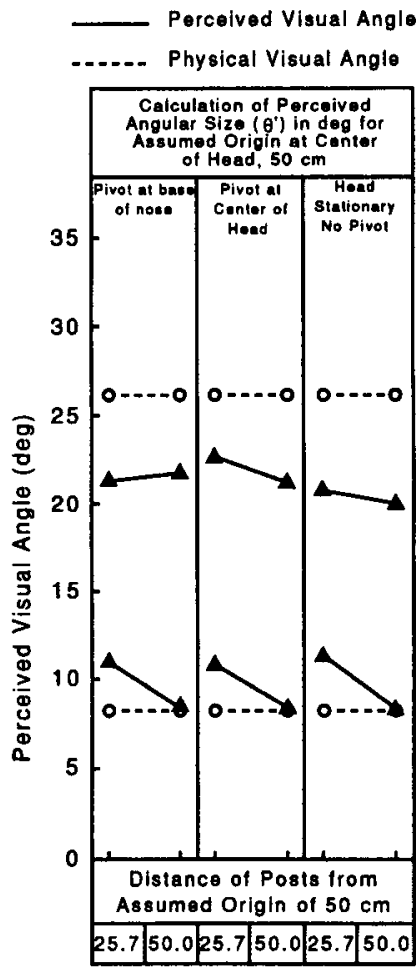

C

Figure 8. In Experiment 3, when the physical pivot (physical origin) was at the base of the nose (essentially at the distance of the chin or eyes), the physical post distances were 15.7 and $40.0 \mathrm{~cm}$ from this physical origin. When the physical pivot (physical origin) was at the center of the head, the physical post distances were 25.7 and $50.0 \mathrm{~cm}$ from this physical origin. When the head was stationary, there was no physical pivot. Figure $8 \mathrm{~A}$ shows the average open-loop adjusted separations of the posts for each of these positions of the physical pivot. Figure 8B shows the average values of $\theta^{\prime}$ computed from the appropriate post separation of Figure 8A, assuming that the perceived origin for all three pivot conditions was at the chin (base of the nose). Figure $8 \mathrm{C}$ shows the average values of $\theta^{\prime}$ computed from the appropriate post separations of Figure $8 \mathrm{~A}$, assuming that the perceived origin for all three pivot conditions was at the center of the head. Clearly, the data of Figure 8C rather than Figure 8B show much smaller deviations in the computed values of $\theta^{\prime}$ as a result of differences in the distance of the posts $(N$, near, and $F$, far) using either the smallest or the largest stimulus.

used in calculating $\theta^{\prime}$ regardless of whether the actual physical distance of the origin was at 40 or at $50 \mathrm{~cm}$ or was unspecified by head rotation in the stationary-head condition. Thus, the term "assumed origin" used in Figures $8 \mathrm{~B}$ and $8 \mathrm{C}$ refers to a hypothesized perceived origin that, if consistent with Experiments 1 and 2, is expected to be more nearly at the distance of $50 \mathrm{~cm}$ from the stimulus used in Figure $8 \mathrm{C}$ rather than the $40 \mathrm{~cm}$ from the stimulus used in Figure 8B. The open circles in Figure 8A give the post separations expected at the near and far distances of the posts if the separations had been physically accurate. The open circles in Figures $8 \mathrm{~B}$ and $8 \mathrm{C}$ give the physical values of $\theta$ calculated from the physical width of the stimulus and a physical origin at $40 \mathrm{~cm}$ (Figure $8 \mathrm{~B}$ ) or at $50 \mathrm{~cm}$ (Figure $8 \mathrm{C}$ ). These are the values of $\theta^{\prime}$ required if $\theta^{\prime}$ were veridical.

\section{Lateral Separations of the Posts for Calculating the Perceived Visual Angle, $\theta^{\prime}$}

According to Figure 4, the adjusted lateral separation of the posts is expected to increase as the distance of the posts from the observer is increased. Clearly, this occurred whether the physical pivot of the head (the physical origin) was at the base of the nose ( $40 \mathrm{~cm}$ from the stimulus), at the center of the head $(50 \mathrm{~cm}$ from the stimulus), or was unspecified in the stationary-head condition, as shown by the slant of the data lines from both the small and large stimuli in Figure 8A. Also, it will be noted that the post adjustments were essentially identical whether the physical origin of the visual angle was provided by the rotating head being at the base of the nose, at the center of the head, or unspecified when the head was stationary. According to Figure 4, at least at the near post distance, 
the post separation was expected to be less when the pivot was at the base of the nose than when the pivot was at the center of the head. However, the essentially identical results shown in Figure 8A for the same size of stimulus indicate that whether the physical origin was at the base of the nose, the center of the head, or unspecified had little or no effect on the adjusted separation of the posts. The perception of the distance of the origin was essentially the same in all three conditions. However, this does not specify whether this perceived origin was at the base of the nose or at the center of the head or somewhere else. To identify the common perceived origin in these three conditions, the following criterion was used. This criterion, as used in Experiments 1 and 2, was that the effective origin for the perception of visual angle (for a particular stimulus size) is that origin at which the measurement of the perceived visual angle (for that stimulus size) remains unchanged as the distance of the measuring posts from the observer is changed. As will be discussed, data related to this criterion are shown in the comparison of Figures $8 \mathrm{~B}$ and $8 \mathrm{C}$.

An ANOVA was performed on the post separation data summarized in Figure 8A using the variables of origin distance $(40 \mathrm{~cm}, 50 \mathrm{~cm}$, or unspecified in the case of the stationary head), stimulus size (large or small), and post distance (near or far). Only the stimulus size and post distance and their interactions were significantly different $[F(1,15)=131.46, p=.00 ; F(1,15)=108.73, p=.00$; and $F(1,15)=103.80, p=.00$, respectively]. Neither the factor of origin nor its interactions with any of the other factors reached statistical significance at the .05 level.

\section{Calculations of Perceived Visual Angle}

Figures $8 \mathrm{~B}$ and $8 \mathrm{C}$ together have a structure similar to that of Figure 5 . The data of Figures $8 \mathrm{~B}$ and $8 \mathrm{C}$ are average values of $\theta^{\prime}$ calculated using the lateral separation of the posts summarized in Figure 8A and the physical distances of the posts from either a near $(40 \mathrm{~cm})$ or far $(50 \mathrm{~cm})$ physical origin, respectively. Thus, for the near $(40 \mathrm{~cm})$ distance of the physical origin, $\theta^{\prime}$ was calculated using the lateral separations of the posts in Figure 8A and the physical distances of 15.7 (near) and $40.0 \mathrm{~cm}$ (far) of the posts from this origin. For the far $(50 \mathrm{~cm})$ distance of the physical origin, $\theta^{\prime}$ was calculated using the same lateral separations of the posts from Figure $8 \mathrm{~A}$ but with the physical distances of 25.7 (near) and $50.0 \mathrm{~cm}$ (far) of the posts from this origin. As noted in the results found in the three columns of Figure $8 \mathrm{~B}$, a near physical origin $(40 \mathrm{~cm})$ was assumed, and in the three columns of Figure $8 \mathrm{C}$, a far origin $(50 \mathrm{~cm})$ was assumed in the calculations of $\theta^{\prime}$ regardless of whether the physical origin was at 40 or $50 \mathrm{~cm}$ from the stimulus. Thus, if the observer in all conditions always perceived the origin of the visual angle to be at $50 \mathrm{~cm}$, applying an origin of $50 \mathrm{~cm}$ when calculating the perceived visual angle should increase the similarity of the computed $\theta^{\prime}$ as a function of post distance over that obtained from applying an origin of $40 \mathrm{~cm}$. This should occur whether the physical location of the pivot was at 40 or $50 \mathrm{~cm}$ from the stimulus and whether the head rotated or was stationary. It is obvious from Figure $8 \mathrm{C}$ for both the upper and lower sets of stimulus data points (from the large and small stimulus, respectively) that this occurred. Assuming the origin to be at $50 \mathrm{~cm}$ (the center of the head) rather than $40 \mathrm{~cm}$ (the base of the nose), produced measures of $\theta^{\prime}$ from the same physical size of stimulus that were more nearly constant (more independent of post distance), regardless of whether the physical origin was at the base of the nose or at the center of the head and regardless of whether the head was rotated or stationary. This result as occurring from the stationary head was somewhat unexpected since the location of the cyclopean eye from which perceived direction is determined often is assumed to be closer to the eyes of the observer than to the center of the head (see Mitson, Ono, \& Barbeito, 1976; Ono, 1991). According to the data of Figure 8 (Experiment 3 ) and Figure 5 (Experiments 1 and 2 ), the observer tends to perceive the origin of a perceived visual angle as being behind the eyes (perhaps at approximately the center of the head) regardless of the physical distance of the pivot or of whether the head is stationary or rotating.

ANOVAs were applied to a comparison of the data of Figures $8 \mathrm{~B}$ and $8 \mathrm{C}$. The variables used were the assumed origin $(40$ or $50 \mathrm{~cm})$, stimulus size (large or small), and post distance (near or far). All three of these variables in each of the three sets of column comparisons were statistically significant at, at least, the .03 level, as were all the first order interactions. Second order interactions also were statistically significant at, at least, the .01 level in comparing the $40-$ and $50-\mathrm{cm}$ distances of the assumed origins for the pivot at the base of the nose and the center of the head in the conditions of head rotation but not for the head-stationary condition, for which the significance level reached only a $p$ of .07 . The comparisons of interest in evaluating the similarity of $\theta^{\prime}$ from the near and far post distances are the first and second order interactions involving the $40-$ and $50-\mathrm{cm}$ assumed origins. As suggested by the results and analysis, of the two physical origins of 40 and $50 \mathrm{~cm}$, the one that produced the greatest similarity in the measurement of $\theta^{\prime}$ for the near and far post distance was the origin at $50 \mathrm{~cm}$.

\section{Discussion}

Two kinds of errors in the measurement of the perceived visual angles are evident in the present study. One is the tendency for the measurement of the average perceived visual angle of the small stimulus $\left(\theta_{\mathrm{s}}^{\prime}\right)$ to be equal to or to exceed its physical visual angle, whereas the measurement of the average perceived visual angle of the large stimulus $\left(\theta_{\mathrm{L}}^{\prime}\right)$ is usually less than its physical visual angle (see Figures $3 \mathrm{C}, 6 \mathrm{C}$, and $8 \mathrm{C}$ ). These results are considered to be in error rather than reflecting accurate measures of $\theta^{\prime}$ because of their inability in Experiments 1 and 2 to predict (using Equation 4 ) the perceived direction of motion in depth from an optical expansion or contraction. It does not seem, however, that this kind of error in measuring $\theta^{\prime}$ can be attributed to errors in the 
kinesthetic sensing of the post separations since, in Experiments 1 and 2 (see Figures $3 \mathrm{~A}$ and $6 \mathrm{~A}$ ), there is no evidence in the present study for systematic errors in the kinesthetic separation of the posts at the different distances when measuring perceived linear extent. This is consistent with evidence from Gogel, Wist, and Harker (1963, Figure 6) and from Teghtsoonian and Teghtsoonian (1965). However, the possibility remains (see Cheng, 1968; Davidon \& Cheng, 1964) that if the perceived kinesthetic distances of the posts from the observer for the measurement of $\theta^{\prime}$ did not equal their physical distances, the use of the physical distances in computing $\theta^{\prime}$ would introduce error. Such differences between physical distance and perceived kinesthetic distance would be unimportant in the measurement of perceived linear size, $S^{\prime}$, since this measure, again as indicated in Figures 3A and $6 \mathrm{~A}$, was independent of the perceived or physical distance of the hands from the body. More generally, the visually perceived size-distance relation involved in the computation of $\theta^{\prime}$ can be tested adequately using kinesthetic adjustments only if the kinesthetic adjustments satisfy the same perceived size-distance relation. Perhaps the kinesthetic adjustments did not meet this requirement.

The second, quite different, source of error in the measurement of perceived visual angle results from errors in perceiving the distance position (origin) from which the change in direction to the ends of the stimulus (the origin of the perceived visual angle) is being made. It is clear from the results that the distance of the origin that should be used in calculating the perceived visual angles in the present study is more nearly at $50 \mathrm{~cm}$ rather than $40 \mathrm{~cm}$ from the stimulus. However, there is a probable complication in locating a single cyclopean origin at about the center of the head in the present study. As calculated from an origin at the center of the head, the average values of $\theta^{\prime}$ for the small stimulus in Experiments 1 and 2, unlike those from the large stimulus (see Figure 5), decreased as the post distance increased. Also, in Experiment 3, using the $50-\mathrm{cm}$ origin, the decrease in $\theta^{\prime}$ for the small stimulus exceeded that for the large stimulus (see Figure 8C) as the post distance increased. This suggests that the perceived origin for the small stimulus was located at a greater distance behind the eyes than was the perceived origin for the large stimulus. In this case, different origins would be needed when using differently sized stimuli to achieve consistent measures of $\theta^{\prime}$ as a function of post distance. If the differences in the perceived origins for $\theta_{\mathrm{L}}^{\prime}$ and $\theta_{\mathrm{s}}^{\prime}$ are valid, these origins may provide the cyclopean distances for judging perceived distance as well as perceived direction. It could follow that differences in the origins as a function of stimulus size could explain the larger perceived distance of the small static stimulus compared with the large static stimulus found in Experiments 1 and 2, as can be calculated from Equation 1. Finally, it should be noted that the perceived location of the origin is important not only for measuring the perceived visual angle, particularly for stimuli relatively near the observer, but also for measuring the perception of direction. According to this interpretation, it would seem that the per- ception of direction and perhaps perceived distance as well as perceived visual angle would differ depending on the perceived distance of the origin from the stimulus.

\section{GENERAL DISCUSSION}

\section{Interrelation of $S^{\prime}, \theta^{\prime}$, and $d^{\prime}$}

Although not always statistically significant, the perceived sagittal motion results in Experiment 1 tended to be larger than those in Experiment 2, and the obtained perceived linear size and perceived visual angle in Experiment 2 tended to be larger than those in Experiment 1 . However, in general, the results from the two experiments are not greatly different, suggesting that similar processes are responsible for the perceptions in both. Thus, the results from Experiments 1 and 2 can often be considered together.

A main result from Experiments 1 and 2 is that the small amount of size constancy present was inappropriate to the large amount of sagittal motion in depth that was perceived. This conclusion, reflected quantitatively in the inability to accurately predict the amount of perceived sagittal motion obtained using the SDIH as expressed by either Equation 2 or 4 in the present study, is supported by the study of Swanston and Gogel (1986), in which a large perceived motion in depth was obtained in the absence of any positive amount of size constancy. These are not the usual conclusions from situations in which no size or distance information other than the optical expansion or contraction is available to possibly determine both the perceived size and perceived motion in depth of the stimulus. Usually (but not always) in such situations, it is reported that either perfect (complete) or approximately perfect size constancy is present. Or more generally, it is concluded that the amount of size constancy is consistent with the perception of the magnitude of motion in depth (see Johansson, 1977, for a discussion of much of this evidence).

Often in studies of optical expansion or contraction, information as to the amount of size constancy and the perceived motion in depth is obtained from the observer's verbal reports. A possible confusion between subjective (apparent) and objective (physical) judgments might have contributed to the difference in the results from these studies and from the linear size adjustments obtained in the present study or in the study of Swanston and Gogel (1986). Two factors can be dismissed as providing possible explanations for the lack of substantial amounts of size constancy obtained in the present study. One is that the measure of perceived linear size was actually a measure of perceived angular size. This is not a concern in the present study since the measures of perceived linear size were essentially independent of the distance of the posts (and of the observer's hands), whereas the measures of perceived angular size consistently increased as the posts were more distant from the observer.

The second factor to be rejected in explaining the lack of substantial amounts of size constancy is that the small inappropriate amount of size constancy that was obtained 
could have been compensated for in part or wholly in the dynamic conditions if the difference between the largest and smallest perceived visual angles $\left(\theta_{\mathrm{L}}^{\prime}-\theta_{\mathrm{s}}^{\prime}\right)$, to be applied in Equation 4, sufficiently exceeded that between the largest and smallest physical visual angles $\left(\theta_{\mathrm{L}}-\theta_{\mathrm{s}}\right)$ of the stimulus. This did not occur in the present study. But perhaps this failure can be attributed to errors in measuring $\theta_{\mathrm{L}}^{\prime}$ and $\theta_{\mathrm{s}}^{\prime}$ (i.e., the underestimation of $\theta_{\mathrm{L}}^{\prime}$ relative to $\theta_{\mathrm{s}}^{\prime}$ ), whereas an overestimation of $\theta_{\mathrm{L}}^{\prime}$ relative to $\theta_{\mathrm{s}}^{\prime}$ is needed if Equation 4 is to fit the dynamic $d^{\prime}$ data. A procedure that is relatively independent of such errors is to compare $\theta_{\mathrm{L}}^{\prime}-\theta_{\mathrm{s}}^{\prime}$ in the static and dynamic conditions in Experiments 1 and 2. The overall average differences in $\theta_{\mathrm{L}}^{\prime}-\theta_{\mathrm{s}}^{\prime}$ from Experiments 1 and $2\left(11.9^{\circ}\right.$ from the static and $13.0^{\circ}$ from the dynamic conditions) are quite similar, as is indicated also in the data of Figures $3 \mathrm{C}$ and $6 \mathrm{C}$, respectively, using an origin at $40 \mathrm{~cm}$ from the stimulus. However, the perceived difference in the distance between the terminal dynamic stimuli was likely to have been greater than the perceived difference in the distance between the static presentations. In other words, even though inaccuracies occurred in measuring the values of perceived visual angle, the differences between $\theta_{\mathrm{L}}^{\prime}$ and $\theta_{s}^{\prime}$ would be expected to be greater in the dynamic than in the static situations of Experiments 1 and 2, if there was a tendency for the visual system to compensate for the small amounts of size constancy in the dynamic conditions by increasing the difference between the values of $\theta_{\mathrm{L}}^{\prime}$ and $\theta_{\mathrm{s}}^{\prime}$ to be used in Equation 4. This likely inability to remedy the predictions from the SDIH by applying Equation 4 rather than Equation 2 to the dynamic conditions, although somewhat tentative, might also apply to other situations such as the moon illusion and the sizedistance paradox, in which the SDIH as expressed by Equations 1 and 2 has seemed to fail (Higashiyama, 1992; McCready, 1965, 1985, 1986). At least the present results suggest that modifications of $\theta^{\prime}$ needed to repair a failure of the SDIH in its usual form (Equations 1 or 2) must be demonstrated by direct measures of $\theta^{\prime}$, rather than by assuming that the required modifications of $\theta^{\prime}$ necessarily occur.

\section{Explaining Results From Experiments 1 and 2}

In Experiments 1 and 2 of the present study or Experiments 1 and 3 of the study by Swanston and Gogel (1986), it seems that the optical changes did not produce responses that modeled any possible physical configuration since the perception of change in linear size and in sagittal motion was not consistent with either the change in physical visual angle, as required by Equation 2, or the change in perceived visual angle, as required by Equation 4 . How is this internal inconsistency to be understood? It has been suggested (Gogel, 1990) that such inconsistencies might reflect the intrusion of cognitive processes into perceptual processes. Swanston and Gogel argued that a cognitive process termed "off-sized perceptions" can be involved in optical expansions or contractions as a result of the rapid or continuous changes in visual angle. Off- sized perceptions or off-sized judgments are perceptions or judgments of the size of the stimulus compared with the size of some previous or standard stimulus and are defined as $S^{\prime} / S_{\mathrm{c}}$, where $S^{\prime}$ is the immediately present stimulus and $S_{\mathrm{c}}$ is the standard (comparison) stimulus from some prior presentation. For example, as compared with the more usual (standard) moon near the zenith, the moon on the horizon appears unusually large (a large off-sized object). Or compared with a normal (standard) playing card, a half-sized playing card at the same perceived distance will appear to be a small off-sized object. Conversely, if the object appears larger or smaller than its normal (standard) size, it often will be reported verbally to be at a nearer or farther distance, respectively, than the distance at which it appears (Gogel, 1969; Gogel \& Newton, 1969). Or, as expressed in equation form, an off-sized judgment or off-sized perception ${ }^{3}$ results in distance responses $\left(D_{\mathrm{c}}\right)$ different from perceived distance, $D^{\prime}$, whenever $S^{\prime} / S_{\mathrm{c}}$ is not equal to 1 , such that

$$
D_{\mathrm{c}}=D^{\prime}\left(S_{\mathrm{c}} / S^{\prime}\right) \text {. }
$$

For example, if an object of normal (customary) size at $20 \mathrm{ft}$ from the observer appears at a distance of $5 \mathrm{ft}$ (for whatever reason), thereby producing the perception of a small off-sized object, the observer will often report the distance of the object as being at or near the correct distance of $20 \mathrm{ft}$. In this case, the off-sized perception has resulted in a modification of the report of the object's distance without producing any modification of its perceived distance; that is, the perception of the object as being at the distance of $5 \mathrm{ft}$ remains unchanged. It will be noted that the report of distance, although not consistent with the perceived distance, provides cognitive information (of about $20 \mathrm{ft}$ ) that is physically more accurate than the perceived distance $(5 \mathrm{ft})$. A variety of studies have indicated that single presentations of objects that appear off-sized, as in the above example, although clearly modifying the verbal reports of distance, are severely limited in modifying or are unable to modify the perception of depth or distance (see, e.g., Gogel, 1976, 1981; Gogel \& Da Silva, 1987; Predebon, 1994a, 1994b; Predebon \& Woolley, 1994).

In the optical expansion and contraction in Experiments 1 and 2 of the present study, continually changing off-sized perceptions were present. In the optical expansion, the object of a constant identity appeared to grow larger as the object appeared to move toward the observer. In the optical contraction, the object of a constant identity appeared to become smaller as it appeared to move away from the observer. The evidence from the study of Swanston and Gogel (1986), however, is that the reported sagittal motion from a repetitive expansion and contraction is a perceived and not simply a cognitive judgment. This evidence was obtained from using the lateral head motion procedure (see Gogel, 1976) to measure the perceived distance of the terminal stimuli of the optical changes. It is proposed that this measurement procedure would not have indicated a change in perceived depth if the change had remained only cognitive, not per- 
ceptual. It follows that an explanation of the robust perceived sagittal motion obtained in the present Experiments 1 and 2 in terms of continuous changes in off-sized perceptions is an assertion that (unlike the results from presenting a static off-sized perception) the presentation of dynamic off-sized perceptions can intrude on perceptual processes, increasing the perception of sagittal motion beyond that expected from Equation 2 of the SDIH.

Additionally, Swanston and Gogel (1986) found that off-sized perceptions in dynamic conditions can act as an independent factor even in the case in which some normally effective cue of changing distance is available. For example, in Experiment 2 of that study, consistent with Emmert's Law (Emmert, 1881), changing the perceived distance of a line stimulus of constant visual angle using only changes in convergence, as expected, resulted in changes in the perceived size and distance of the stimulus. The changes in perceived size, however, provided offsized perceptions whose effect, opposite to that from the convergence, was such that it reduced the perceived depth obtained from the convergence changes. This result was demonstrated by the greater perceived depth from the same convergence changes obtained when a point of light rather than an extended object was the stimulus used. More generally, it was also found using an extended object and changing perceived sizes that off-sized perceptions in agreement or in opposition to the effect of convergence changes could add or subtract, respectively, from the perceived depth produced by the convergence alone. Also, Experiment 4 of the Swanston and Gogel study identifies an additional property that dynamic off-sized perceptions have in common with the usual cues of distance. This is an increase in the perceived depth from a given change in distance cues when the same change occurs at an increased distance from the observer (Gogel, 1964; Ono \& Comerford, 1977; Ono, Rivest, \& Ono, 1986; Wallach \& Zuckerman, 1963). Although this phenomenon (sometimes called "the inverse square law"), obtained from using changes in off-sized perceptions, was not large, it occurred in Experiment 4 of Swanston and Gogel at the .05 level of significance.

Such results add to the evidence that, particularly when the usual cues of distance are somewhat limited, offsized perceptions can modify the perception of depth or distance in dynamic conditions in a manner equivalent to the role of the more conventional cues of depth or distance. However, off-sized perceptions in dynamic conditions differ from the more conventional cues of perceived distance in that they can occur only when the more conventional cues provide initial perceptions of size and distance. Thus, in the beginning of the repetitive optical changes in the present study, the perception of the stimuli as off-sized was dependent on the presence of information regarding perceived egocentric distance, in this case determined by cues of constant accommodation and accommodative convergence, and aided by the specific distance tendency. These factors (called "primary factors"), essential for perceiving the changes in angular size as changes in linear size (i.e., factors essential for seeing the stimuli as off-sized perceptions), not only provide the possibility of the presence of off-sized perceptions (called "secondary factors") but probably also can restrict partially or totally the contribution to the perception of sagittal motion provided by off-sized perceptions. For example, in Experiments 1 and 2 of the present study, if the constant cues of distance provided for instance by accommodation had been sufficiently effective in determining perceived distance, the observers would have perceived the stimulus as increasing in linear size proportional to its increase in angular size, but also as remaining throughout the optical change at a constant distance from the observer (zero perception of sagittal motion). Or, on the other hand, if changing primary cues (e.g., the relative size cue occurring between the succeeding presentations in Experiments 1 and 2) had been more effective in the present study, the ability of Equation 2 to predict the amount of perceived sagittal motion would have increased, and the contribution of the secondary cues would have been reduced or eliminated. Thus, the primary cues of distance are both essential (in the case of unchanging primary cues) and restrictive (in the case of both unchanging and changing primary cues) for the ability of the secondary cues to modify the perception of sagittal motion.

Although the effect of off-sized perceptions on perceived distance provides a reasonable explanation of the $d^{\prime}$ not predicted by Equation 2 in the present study, the understanding of the process by which their contribution is accomplished is not complete. Probably the major problem of applying off-sized perceptions to explain the results obtained in Experiments 1 and 2 of the present study is in identifying the standard (comparison) size against which the magnitude of the momentary off-sized characteristic is determined. All off-sized perceptions require an internal representation of a standard size determined by a prior perception of size or perhaps by some other prior perceptual source of size information. This comparison size is a cognitive size $\left(S_{\mathfrak{c}}\right)$ since it involves the internal storage and retrieval of a prior representation of the object. It would be convenient to assume that the standard size throughout an optical expansion or contraction remains the size determined by the starting size of the optical change. But this, particularly when a series of complete cycles of contraction and expansion is used, is unlikely. As the cycles of expansion and contraction continue and as the number of cycles of repetitions increases, the standard size, for example, might shift toward the average of the presentations. Also, in Experiments 1 and 2 of the present study, the amount of size constancy as applied in Equation 2 can explain about one half of the $d^{\prime}$ obtained, thereby limiting the need to attribute the role of off-sized perceptions in little more than the remaining half of the perceived sagittal motion, thus complicating the identification of the comparison size or sizes needed to account for the contribution of the offsized perceptions to the perceived sagittal motion.

As noted in the above discussion, off-sized perceptions tend to make the distance response of the observer more accurate with respect to the physical world by countering 
any errors from primary cues that occur in the perception of distance. For example, the perception that a distant person looks smaller in perceived size than a nearby person of the same physical size provides information that the distant person is indeed far away. Of consequence for the present study, the effect of the continuous changes in off-sized perceptions by contributing to the production of perceived sagittal motion tends to make the perceived sagittal motion at least partially independent of the amount of size constancy present.

The overall amount of size constancy available from the static presentations averaged over Experiments 1 and $2(.365)$ is very nearly the same as that averaged over the dynamic optical changes (.366). The dynamic presentations contributed little or nothing to the size constancy beyond that provided by the static presentations. In both the static and dynamic conditions, this amount of size constancy is small and, in the dynamic conditions, was inappropriate to the amount of sagittal motion perceived. Perhaps the presence of any amount of size constancy in Experiments 1 and 2 can be attributed to the relative size cue occurring between the successive presentations. According to this explanation, it is as though the observer assumes that objects of the same shape are more similar in size than would be expected if their shapes had differed. This possibility, however, would not result in a quantitative prediction of the amount of size constancy expected. Another possible source of this constancy effect is found in the evidence indicating that the origin of $\theta^{\prime}$ for the small stimulus is farther behind the eyes than that for the large stimulus, thereby making the difference in their perceived sizes smaller than the difference in their physical sizes. The latter interpretation depends on the assumption that the visual perception of distance, like the visual perception of direction, is processed from the position of the cyclopean eye.

In summary, it is proposed that part of the changes in visual angle were utilized to produce the perceptions of size relative to some previous presentation in the optical change providing a comparison size, and that the resulting off-sized perceptions in turn produced a substantial part of the perception of sagittal motion. As discussed previously, unlike the effects from static off-sized presentations, evidence is available (Swanston \& Gogel, 1986, Experiment 3 ) that the judgments of sagittal motion in the dynamic conditions were indeed perceptions. If so, the study by Swanston and Gogel and the present study illustrate that a cognitive factor, $S_{\mathrm{c}}$, in the off-sized perception $\left(S^{\prime} / S_{\mathrm{c}}\right)$ can contribute to the perception of sagittal motion, thereby producing or augmenting the perceived motion in depth even if the usual cues of changing distance are completely absent and size constancy is unavailable or inadequate. Perhaps this process occurs because it has a high degree of ecological validity in perceiving motion in distance. An object that is perceived to systematically change in linear size while remaining essentially constant in shape or identity, whether or not any size constancy is available, is unlikely to be an object that is remaining at a constant distance from a stationary observer.

\section{Summary and Concluding Remarks}

It is often assumed that complete or nearly complete size constancy will occur from an optical expansion or contraction that is presented under conditions in which essentially only the optical change is available to determine changes in perceived size and distance (see Hershenson, 1992a, 1992b; Johansson, 1977). The present study does not support this assumption. In addition, according to the present results, although a robust perception of motion in depth was obtained, only a very limited amount of sizeconstancy was available. Such results are inconsistent with Equation 2 of the SDIH in that only about half of the sagittally perceived motion was predicted from this equation. Also, this failure of prediction was not rectified by using measured (perceived) visual angles of the stimuli in Equation 4 of the SDIH rather than the physical visual angles of the stimulus used in Equation 2. Nor can this predictive error be explained totally by errors in measuring perceived visual angle, since the measured visual angle clearly increased as information indicating an increase was provided by the physical change in the angular size of the stimulus.

The procedure used in measuring perceived angular size in order to contrast it with perceived linear size, as illustrated in Figure 1, produced an unexpected result. It was found, for a stimulus of constant size at a constant distance from the observer, that the measured value of perceived angular size was not always independent of the distance of the measuring posts from the observer. A criterion was used for investigating the functional origin of the perception of visual angle. It was hypothesized that the functional (perceived) origin for measuring the perceived visual angle was the distance from the stimulus at which the perceived visual angle was essentially the same as that measured at the different distances of the posts. Applying this criterion, it was evident that this effective (perceived) origin was located at a distance considerably behind the eyes regardless of whether the actual (physical) pivot distance from the stimulus was at this distance or not. This functional (perceived) origin was found to be essentially the same whether the head remained stationary or was turned to point the nose alternately at the left or right direction of the sides of the stimulus. In addition, however, the results from all three experiments suggest that this perceived origin was further from the stimulus when the smallest rather than the largest angular stimulus was measured. For this there does not seem to be any ready explanation. These findings are of interest regarding the position of the functional (cyclopean) eye and its relation to perceived distance and perceived visual angle as well as to perceived direction.

A physical object of constant angular size necessarily changes in visual angle as it physically moves toward (or away from) the observer. If effective (accurate) cues of the changing distance of the stimulus are available, the 
object is likely to remain constant in perceived linear size throughout its physical motion. Since the size constancy is perfect, no off-sized perceptions are present and Equation 2 can be expected to completely predict the perceived sagittal motion $\left(d^{\prime}\right)$. If this happens, the configuration of responses obtained will model or be modeled by the physical configuration being viewed. A situation that produces the same changes in physical visual angle as would be obtained with an object of constant physical size moving in physical sagittal distance can be achieved by presenting the appropriate stimulus changes in linear size on a monitor screen at a constant distance from the observer. Such a situation, simulating an optical expansion, is illustrated in Figure 9A. If effective cues of changing distance of the stimulus in this simulation are available, the stimulus will appear constant in linear size and, by definition, no off-sized perceptions will be present. In this case, Equation 2 will completely predict the $d^{\prime}$ obtained, and the configuration of responses will model or be modeled by the physical situation being simulated. However, if only changes in physical visual angle are available - that is, if effective cues of changing distance

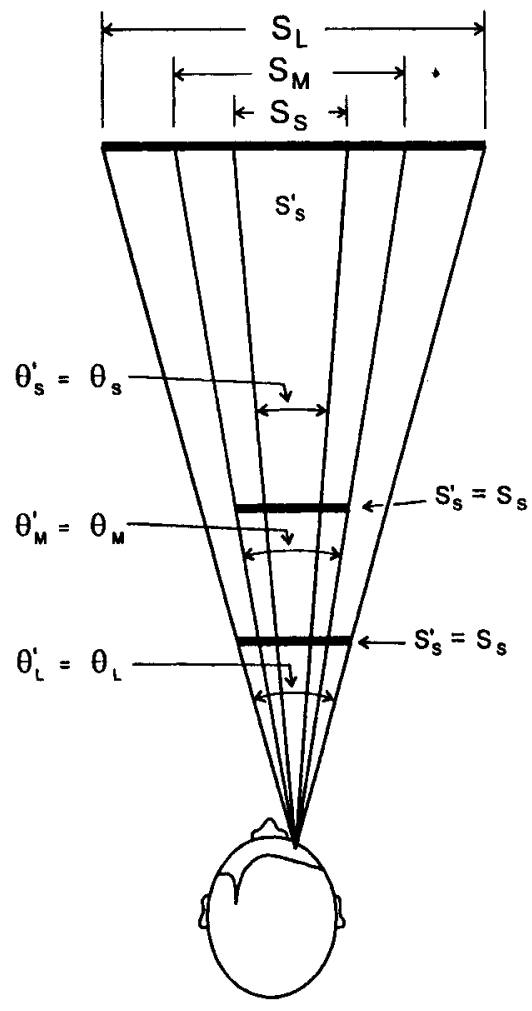

Obs.

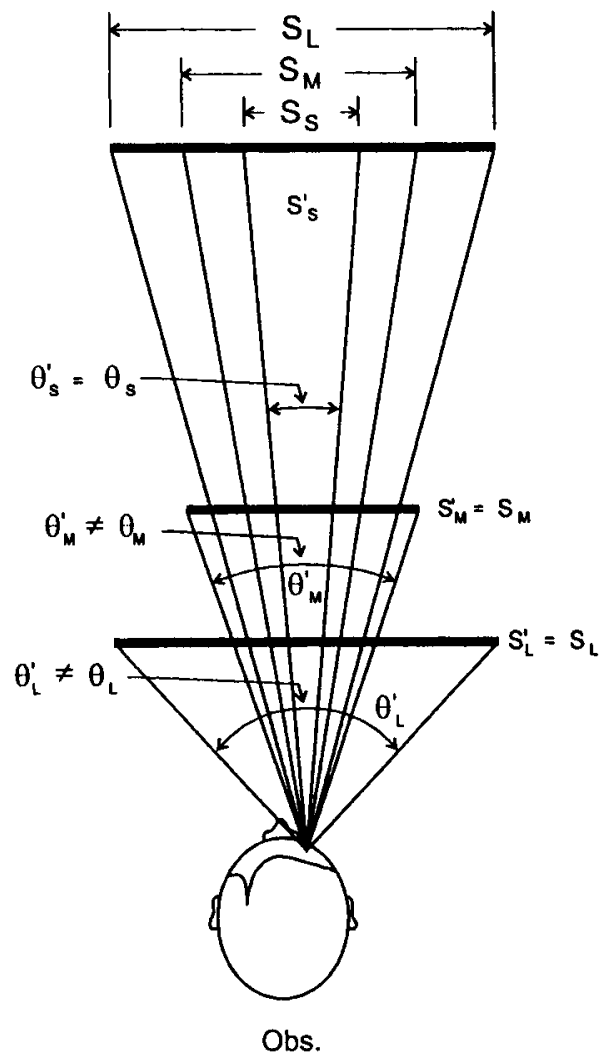

A

Figure 9. This figure illustrates conditions in which the size-distance invariance hypothesis (SDIH) is able to predict the total perceived sagittal motion $\left(d^{\prime}\right)$ produced in this illustration by an optical expansion. The stimulus changes in both drawings are identical and are shown as generated on a monitor by a square stimulus expanding in width from $S_{\mathrm{S}}$ to $S_{\mathrm{M}}$ to $S_{\mathrm{L}}$. As the stimulus appears to move toward the observer in Figure $9 \mathrm{~A}$, it remains constant in perceived size (a simulation of perfect size constancy), whereas in Figure $9 B$ it appears to increase in size with each size increase on the monitor (a simulation of zero size constancy). Equation 2 would predict all of the perceived motion in depth in the situation of Figure 9A, and Equation 4 would predict all of the perceived motion depth in the situation of Figure 9B, but, in the latter case, only if the perceived visual angles increased at a faster rate than the physical visual angles produced on the monitor. Neither of these drawings separately apply to the results obtained in Experiments 1 and 2 of the present study. That is, the perfect size constancy in order for Equation 2 to apply fully to Figure $9 \mathrm{~A}$ and the increased perceptual enlargement of the visual angles of the stimulus in Figure 9B compared with Figure 9A did not occur. Complete size constancy, as shown in Figure 9A, probably can be found only under conditions in which very effective cues of changing distance are present, and the necessary angular enlargements illustrated in Figure 9B have yet to be demonstrated. Thus, in Experiments 1 and 2 , a cognitively determined component of $d^{\prime}$ from off-sized perceptions, together with a limited contribution from Equation 2 of the SDIH, is needed to explain the results. 
are absent, throughout the optical change, as in Experiments 1 and 3 of the Swanston and Gogel (1986) study, or are only partially available, as in Experiments 1 and 2 of the present study-perfect size constancy will not be present. In such cases, off-sized perceptions will be present determining or contributing to the perception of the sagittal motion of the stimulus and Equation 2 will not predict the full $d^{\prime}$ perceived. Equation 2 can be expected to predict the total sagittal motion perceived in an optical expansion (or contraction) only when the cues of changing perceived distance are sufficiently effective so as to produce complete size constancy, defined by $S_{\mathrm{s}}^{\prime} / S_{\mathrm{L}}^{\prime}=1$.

In Figure 9B, the perception of linear size, instead of remaining constant as in Figure 9A, increases proportionately with the increase in the stimulus size on the monitor, thereby producing zero size constancy and clear offsized perceptions. The off-sized perceptions in this case are considered to produce a perception of sagittal motion similar to that obtained with the effective cues of changing distance in Figure 9A. In order for the SDIH, using Equation 4, to predict the full amount of depth perceived in Figure 9B, the visual angles as perceived must increase considerably more rapidly than the physical visual angles presented on the monitor. But such a result as that diagrammed in Figure 9B was not obtained in the present study. Thus, it seems that the SDIH often must be considered as only one of two processes determining the perceptions associated with an optical expansion or contraction, with the second process consisting of a cognitive process specified by off-sized perceptions. A consequence of the perceived visual angles failing to increase in angular size-as required by Figure $9 \mathrm{~B}$ if Equation 4 is to predict the $d^{\prime}$ obtained-is that the configuration as perceived simulates an object that increases in physical size as it moves toward the observer while failing to increase appropriately in angular size. But that is physically impossible. Such a result would neither model nor be modeled by any physical configuration, indicating that not all of the response variables $\left(S^{\prime}, d^{\prime}, \theta\right.$, or $\left.\theta^{\prime}\right)$ are consistent with each other. Such inconsistencies are, however, characteristic of the influence of a cognitive process (see Gogel, 1990, Figure 9), here specified as off-sized perceptions, that has contributed in the present study to the perception of the sagittal motion of the stimulus in optical expansions or contractions.

The present study together with the Swanston and Gogel (1986) study support the following implications or conclusions:

1. The perception of sagittal motion produced in optical expansions or contractions in which changes in angular size is the only variable present to determine both perceived linear size and perceived sagittal motion is likely to differ substantially from that obtained in situations in which effective cues of changing distance are available. The former compared to the latter situation is likely to have more perceived sagittal motion determined by offsized perceptions (less size constancy) and to show less ability of the SDIH to predict the $d^{\prime}$ obtained.
2. The less the amount of size constancy in dynamic conditions (the greater the amount of off-sized perceptions), the less likely it is that the configuration perceived will model any physical configuration and the less likely it is that all the variables in the perceptual configuration will be consistent with each other. As shown in the present study, this result cannot be attributed to observer confusion as to the difference between perceived angular and linear extent.

3. The internal inconsistency in the response configuration resulting from off-sized perceptions is an instance of a modification of a perceptual (primary) response (perceived sagittal motion) by a cognitive (secondary) response (off-sized perceptions), as indicated in Experiments 1 and 3 of Swanston and Gogel (1986). However, only in dynamic situations is the modification of the response to perceived distance by off-sized perceptions considered to be substantially perceptual.

4. That off-sized perceptions are a frequent and significant factor in many conditions of optical expansion or contraction is shown by the frequent occurrence of less than perfect size constancy. The off-sized perceptions in these situations, by contributing to the perception of sagittal motion, limit the ability of the SDIH to predict the full amount of $d^{\prime}$ obtained. However, the contribution of the SDIH to the prediction of some of the $d^{\prime}$ obtained in all situations in which the size constancy is not zero indicates the importance of the SDIH in an explanation of the motion perceived.

5. Off-sized perceptions can be important in a variety of dynamic conditions, as is suggested by their ability to modify the application of Emmert's Law (Emmert, 1881), to modify the effect of the convergence cue of distance, and to comply (at least in direction) with the inverse square law (as noted by Swanston and Gogel, 1986, Experiment 4). This evidence, together with the response inconsistencies between the variables of $S^{\prime}, d^{\prime}$, and $\theta$ or $\theta^{\prime}$ in optical changes in which off-sized perceptions are present, makes it increasingly likely that off-sized perceptions are responsible for the $d^{\prime}$ in optical expansions or contractions that is not predicted from the SDIH. Thus, the question concerning the SDIH is not whether the SDIH is able to predict all the perception of sagittal motion perceived in an optical expansion or contraction. Often it is not, particularly in situations involving some reduction in distance cues. Instead, the problem is to determine the relative contribution of the SDIH in dynamic situations in which cognitive processes such as off-sized perceptions also contribute.

\section{REFERENCES}

BörJesson, E., \& VON Hofsten, C. (1972). Spatial determinants of depth perception in two-dot motion patterns. Perception \& Psychophysics, 11, 263-268

CHENG, M. F. (1968). Tactile-kinesthetic perception of length. American Journal of Psychologv, 81, 74-82.

Davidon, R. S., \& Cheng, M. F. (1964). Apparent distance in a horizontal plane with tactile-kinesthetic stimuli. Quarterly Journal of Experimental Psychology, 16, 277-281. 
Emmert, E. (1881). Grossenverhältnisse der Nachbilder. Klinische Monatsblatt für Augenheilkunde, 19, 443-450.

FOLEY, J. M. (1967). Binocular disparity and perceived relative distance: An examination of two hypotheses. Vision Research, 7, 655-670.

FOLEY, J. M. (1968). Depth, size and distance in stereoscopic vision. Perception \& Psychophysics, 3, 265-274.

Gillam, B., \& Chambers, D. (1985). Size and position are incongruous: Measurements on the Müller-Lyer figure. Perception \& Psychophysics, 37, 549-556.

GOGEL, W. C. (1964). The perception of depth from binocular disparity. Journal of Experimental Psychology, 67, 379-386.

GoGEL, W. C. (1969). The effect of object familiarity on the perception of size and distance. Quarterly Journal of Experimental Psychology, 21, 239-241.

GOGEL, W. C. (1971). The validity of the size-distance invariance hypothesis with cue reduction. Perception \& Psychophysics, 9, 92-94

GoGEL, W. C. (1976). An indirect method of measuring perceived distance from familiar size. Perception \& Psychophysics, 20, 419-429.

GOGEL, W. C. (1981). The role of suggested size in distance responses. Perception \& Psychophysics, 30, 149-155.

GoGEL, W. C. (1990). A theory of phenomenal geometry and its applications. Perception \& Psychophysics, 48, 105-123.

GogeL, W. C., \& DA Silva, J. A. (1987). Familiar size and the theory of off-sized perceptions. Perception \& Psychophysics, 41, 31 8-328.

Gogel, W. C., \& NEwTON, R. E. (1969). Perception of off-sized objects. Perception \& Psychophysics, 5, 7-9.

GoGEL, W. C., Wist, E. R., \& HARKER, G. S. (1963). A test of the invariance of the ratio of perceived size to perceived distance. American Journal of Psychology, 76, 537-553.

HeRshenson, M. (1992a). The perception of shrinking in apparent motion. Perception \& Psychophysics, 52, 671-675.

Hershenson, M. (1992b). Size-distance invariance: Kinetic invariance is different from static invariance. Perception \& Psychophysics, 51, 541-548.

Hershenson, M. (1993). Structural constraints: Further evidence from apparent motion in depth. Perception, 22, 323-334.

HigashiYama, A. (1992). Anisotropic perception of visual angle: Implications for the horizontal-vertical illusion, overconstancy of size, and the moon illusion. Perception \& Psychophysics, 51, 218-230.

HigashiYama, A., \& Shimono, K. (1994). How accurate is size and distance perception for very far terrestrial objects? Function and causality. Perception \& Psychophysics, 55, 429-442.

Johansson, G. (1950). Configurations in event perception. Uppsala, Sweden: Almquist \& Wiksell.

Johansson, G. (1964). Perception of motion and changing form. Scandinavian Journal of Psychology, 5, 181-208.

JoHANSSON, G. (1977). Spatial constancy and motion in visual perception. In W. Epstein (Ed.), Stability and constancy in visual perception: Mechanisms and processes (pp. 375-419). New York: Wiley.

LoOMIS, J. M., \& EвY, D. W. (1987). High-speed 2-D and 3-D animation on the IBM PC/XT/AT. Behavior Research Methods, Instruments, \& Computers, 19, 10-18.

Mack, A., Heuer, F., Villardi, K., \& Chambers, D. (1985). The dissociation of position and extent in Müller-Lyer figures. Perception \& Psychophysics, 37, 335-344.

MCCREADY, D. (1965). Size-distance perception and accommodationconvergence micropsia-A critique. Vision Research, 5, 189-206.

MCCrEADY, D. (1985). On size, distance, and visual angle perception. Perception \& Psychophysics, 37, 323-334.

McCready, D. (1986). Moon illusions redescribed. Perception \& Psychophysics, 39, 64-72.

MeRshon, D. H., \& Lembo, V. L. (1977). Scalar perceptions of distance in simple binocular configurations. American Journal of Psychology, 90, 17-28.
Mitson, L., Ono, H., \& BARBEito, R. (1976). Three methods of measuring the location of the egocentre: Their reliability, comparative locations and intercorrelations. Canadian Journal of Psychology/Revue Canadienne de Psychologie, 30, 1-8

Noguchi, K., \& TaYa, K. (1981). A neglected problem: Kinetic size constancy. Acta Psychologica, 48, 187-194.

ONo, H. (1970). Some thoughts on different perceptual tasks related to size and distance. In J. C. Baird (Ed.), Human space perception: Proceedings of the Dartmouth Conference (Psychonomic Monograph Supplements, Vol. 3, No. 13 [Whole No. 45], pp. 137-218). Austin, TX: Psychonomic Journals.

ONO, H. (1991). Binocular visual directions of an object when seen as single or double. In D. Regan (Ed.), Binocular vision (Vol. 9, pp. 118). New York: Macmillan.

ONo, H., \& Comerford, J. (1977). Stereoscopic depth constancy. In W. Epstein (Ed.), Stability and constancy in visual perception: Mechanisms and processes (pp. 91-128). New York: Wiley.

ONO, H., \& NaKAMIZO, S. (1977). Saccadic eye movements during changes in fixation to stimuli at different distances. Vision Research, 17, 233-238

ONo, M. E., Rivest, J., \& ONo, H. (1986). Depth perception as a function of motion parallax and absolute-distance information. Journal of Experimental Psychology: Human Perception \& Performance, 12, 331-337.

OYama, T. (1974). Perceived size and perceived distance in stereoscopic vision and an analysis of their causal relations. Perception \& Psychophysics, 16, 175-181.

Predebon, J. (1994a). Convergence responses to monocularly viewed objects: Implications for distance perception. Perception, 23, 303-319.

Predebon, J. (1994b). Perceived size of familiar objects and the theory of off-sized perceptions. Perception \& Psychophysics, 56, 238-247.

PREDEBON, J. (1995). Open-loop pointing responses to the location of the vertices of Müller-Lyer figures. Unpublished manuscript.

Predebon, J., \& Woolley, J. S. (1994). The familiar-size cue to depth under reduced-cue viewing conditions. Perception, 23, 1301-1312.

RICHARDS, W. (1969). The influence of oculomotor systems on visual perception (69-1934 TR Massachusetts Institute of Technology). Cambridge, MA: Air Force Office of Scientific Research.

Rock, I. (1983). The logic of perception. Cambridge, MA: MIT Press. Swanston, M. T., \& Gogel, W. C. (1986). Perceived size and motion in depth from optical expansion. Perception \& Psychophysics, 39, 309-326.

Teghtsoonian, M., \& Teghtsoonian, R. (1965). Seen and felt length. Psychonomic Science, 3, 465-466.

Wallach, H., \& ZuCKerman, C. (1963). The constancy of stereoscopic depth. American Journal of Psychology, 76, 404-412.

\section{NOTES}

1. The term $2 \tan \theta / 2$ (or $2 \tan \theta^{\prime} / 2$ ) rather than $\tan \theta$ or $\tan \theta^{\prime}$ is used in equations and, where applicable, in the calculations throughout this study because the stimuli are centered on the observer's geographical median plane at the height of the observer's eye.

2. Seven of the 12 observers in Experiment 1 (and Experiment 2) had participated in two preliminary experiments requiring tasks similar to those of the present study but with different conditions.

3. It is appropriate to call $S_{\mathrm{c}} / S^{\prime}$ either an off-sized perception or an off-sized judgment since it has both a perceptual component $\left(S^{\prime}\right)$ and a memory component $\left(S_{\mathrm{c}}\right)$

(Manuscript received August 15, 1994; revision accepted for publication August 14, 1996.) 